\title{
Identification of Aeroelastic Models for the X-56A Longitudinal Dynamics Using Multisine Inputs and Output Error in the Frequency Domain
}

\author{
Jared A. Grauer ${ }^{1, *}$ and Matthew J. Boucher ${ }^{2}$ \\ 1 NASA Langley Research Center, Hampton, VA 23681, USA \\ 2 NASA Armstrong Flight Research Center, Edwards, CA 93523, USA; matthew.j.boucher@nasa.gov \\ * Correspondence: jared.a.grauer@nasa.gov
}

Received: 28 November 2018; Accepted: 5 February 2019; Published: 22 February 2019

\begin{abstract}
System identification from measured flight test data was conducted using the X-56A aeroelastic demonstrator to identify a longitudinal flight dynamics model that included the short period, first symmetric wing bending, and first symmetric wing torsion modes. Orthogonal phase-optimized multisines were used to simultaneously excite multiple control effectors while a flight control system was active. Non-dimensional stability and control derivatives parameterizing an aeroelastic model were estimated using the output-error approach to match Fourier transforms of measured output response data. The predictive capability of the identified model was demonstrated using other flight test data with different inputs and at a different flight conditions. Modal characteristics of the identified model were explored and compared with other predictions. Practical aspects of the experiment design and system identification analysis, specific to flexible aircraft, are also discussed. Overall, the approach used was successful for identifying aeroelastic flight dynamics models from flight test data.
\end{abstract}

Keywords: aeroelasticity; flight dynamics; X-56A; system identification; output error; parameter estimation; frequency domain; multisine inputs

\section{Introduction}

Efforts to reduce fuel consumption and increase aerodynamic efficiency of aircraft can lead to vehicle designs having long and slender wings. Such airplanes may experience aeroelastic effects in flight, potentially resulting in performance degradation, structural fatigue, or catastrophic loss of aircraft. To mature modeling and control technologies for flexible aircraft and mitigate these effects, the NASA Advanced Air Transport Technology (AATT) Project has been conducting flight research with the X-56A Multi-Utility Technology Testbed (MUTT) aircraft [1], which was purposefully designed to encounter aeroelastic instabilities within its normal flight envelope [2].

For flight dynamics analysis, a useful model for predicting and understanding the behavior of flexible aircraft is the work of Waszak and Schmidt, which was first presented in Reference [3] and elaborated upon in References [4-7]. Their work extended traditional rigid-body flight dynamics models based on quasi-steady stability and control derivatives (for example, Reference [8]) to include a mean-axis reference frame with generalized modal vibration states and aeroelastic derivatives that couple the aerodynamics and the structural deformation. The Waszak-Schmidt model is applicable for small, linear flight variable perturbations and elastic deformations. Similar to standard flight-dynamics models, it assumes quasi-steady aerodynamics; any unsteady effects are lumped into the aeroelastic stability derivatives. It also assumes that the structural deformation of the aircraft can be decomposed into a superposition of orthogonal vibration modes. Despite these limitations, the Waszak-Schmidt 
model is applicable in a large number of cases, particularly the aeroelastic analysis of the X-56A flight test data. Furthermore, the Waszak-Schmidt model is representative of the observed flight motions, including near flutter, and is of relatively low order, making it useful for flight dynamics analyses and feedback control design.

The Waszak-Schmidt model or similar models have been used in system identification analyses to estimate aeroelastic stability and control derivatives from flight test data. In Reference [9], low-order transfer functions are fit to frequency responses computed from flight test data of the XV-15 tiltrotor VTOL aircraft. In References [10-12], state-space models are fit to computed frequency responses for a large transport aircraft, a YAV-8B Harrier, and a mid-sized business jet. In each of these works, it is argued that, because the lowest vibration mode is at least five times the highest rigid-body mode of interest, the stability and control derivatives that couple the elastic motion of the aircraft to the rigid-body equations of motion can be neglected. This is a simplification of the Waszak-Schmidt model, called the hybrid-flexible model, wherein the structural deformations of the aircraft are registered in the sensor measurements but otherwise do not impact the gross motion of the vehicle. This simplified model is also used in Reference [13], where the filter-error approach is used to identify models for the SR-71 aircraft from measured time histories. In Reference [14], the full Waszak-Schmidt model is used to identify a model of a high-performance glider using an output-error analysis in the time domain. In References [15,16], flight data from frequency sweeps and multisine inputs are used with output-only modal identification and frequency response estimation to identify aeroelastic models of a small, unmanned aircraft. Grauer and Boucher [17] proposed a real-time method based on state reconstruction and the equation-error approach in the frequency domain, and demonstrated the method using X-56A flight test data. In Reference [18], a state-space implementation of the Waszak-Schmidt model is used to fit computed frequency response data of the X-56A operating under closed-loop control with input mixing. Additional case studies in the literature, including less recent works, are summarized in Reference [19].

The aforementioned examples of system identification using aeroelastic flight dynamics models are relatively few compared to the existing literature for rigid-body flight dynamics models. One reason for this disparity is the difficulty in accurately identifying aeroelastic models. In particular, the model size can become very large and incorporate many inputs, outputs, states, and unknown model parameters. Because vibration modes of the aircraft structure can occur at relatively high frequencies, faster sampling rates and higher-quality hardware are needed to obtain adequate modeling data. The necessity to excite a broad range of frequencies, to identify responses from many control effectors, and to estimate a large number of model parameters can result in long data records to achieve the requisite level of data information. All these factors result in computationally intensive system identification analyses. Furthermore, the modal states of the aircraft structure are not directly observable but indirectly affect many of the output response measurements. In addition to the mass and inertia properties needed to identify rigid-body models, structural properties such as modal frequencies and modes shapes must be measured, modeled, or estimated from the data. Lastly, many estimation methods are nonlinear and require iteration from good starting values. Although there is a large body of knowledge for stability and control derivatives of conventional aircraft and rigid-body models, the aeroelastic derivatives are less familiar and can require a separate analysis to obtain appropriate values. Each of these characteristics makes the system identification problem more difficult for flexible aircraft models than for rigid-body models.

In this paper, a different approach for testing flexible aircraft and identifying model parameters is discussed. Orthogonal phase-optimized multisine inputs were used to simultaneously excite multiple control effectors on the X-56A over specified bandwidths and with prescribed power spectra. This step led to an efficient flight test maneuver with good data for linear modeling. Using the Waszak-Schmidt model structure, the output-error approach for parameter estimation was applied to fit Fourier transforms of measured output responses. This approach avoided the need for direct state measurements, incorporated data from numerous inputs and outputs over frequency ranges of 
interest, provided physical insights from the frequency-domain perspective, and reduced the size of the optimization problem needed to estimate stability and control derivatives. The method is restricted to linear models describing small perturbations and deformations about a reference flight condition, such as those typically used in a flutter analysis, and needs good starting values for the model parameters, which were provided by a numerical panel code.

This paper is organized as follows. In Section 2, the X-56A aircraft, flight-test instrumentation, and structural characteristics are discussed. Section 3 details the experiment design, including the orthogonal phase-optimized multisine inputs used to excite the aircraft. The Waszak-Schmidt aeroelastic flight dynamics model used for identification is summarized in Section 4, and the output-error approach using frequency-domain data is discussed in Section 5. Then, Section 6 presents the identification results and characteristics of the identified model. Conclusions are drawn in Section 7.

Due to ITAR restrictions associated with the X-56A airplane, numerical values are not given in this paper. However, the approach is general and not specific to this aircraft or configuration. Routines for the experiment design, data analysis, and system identification are from the MATLAB ${ }^{\circledR}$-based software package called System IDentification Programs for AirCraft (SIDPAC) [20].

\section{Aircraft and Flight Tests}

\subsection{X-56A Aircraft}

Figure 1 shows a photograph of the $\mathrm{X}-56 \mathrm{~A}$ and a set of three-view drawings. The aircraft is a tailless, flying-wing configuration with high aspect ratio, swept-back wings and stationary winglets. The wingspan is $28 \mathrm{ft}$ and the weight is between 400 and $500 \mathrm{lbf}$, depending on fuel and ballast. Two JetCat P-400 engines, each capable of providing $90 \mathrm{lbf}$ of thrust, are mounted above the aft section of the center body. The landing gear are arranged in a fixed, tricycle configuration. The aircraft is modular in design, with two center bodies and four sets of interchangeable wings with different degrees of flexibility. There are two control surfaces along the trailing edge of the center body, and four control surfaces along the trailing edge of each wing. These ten surfaces are used in different combinations to generate aerodynamic control moments, augment stability characteristics, alleviate gust responses, suppress aeroelastic vibrations, and excite the aircraft for system identification.

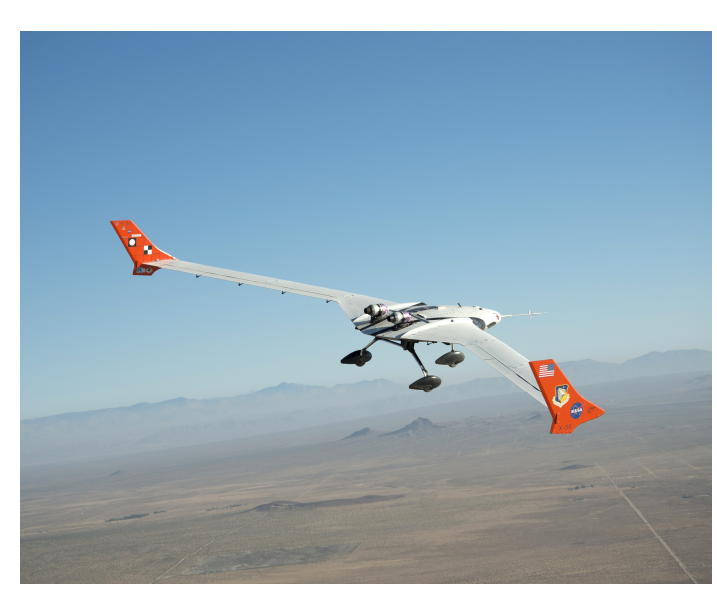

(a)

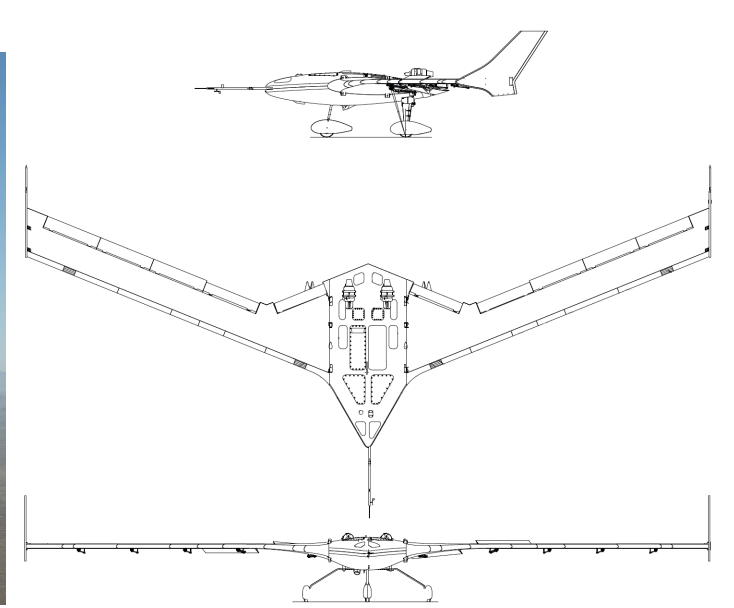

(b)

Figure 1. The X-56A MUTT subscale aeroelastic demonstrator: (a) airplane in flight over NASA Armstrong Flight Research Center (credit: NASA/Jim Ross); and (b) three-view drawing schematic.

The X-56A is flown remotely using a ground control station. Inside the station are seats for a pilot and a co-pilot to fly the aircraft using the stick and rudder controls, an out-the-nose video with overlaid heads-up-display drawn using telemetered data, and a configurable glass-cockpit display. There are 
additional seats for flight test engineers to monitor telemetered data during flight tests. The ground control station also contains a nonlinear simulation of the vehicle that uses the same hardware as to fly the airplane, which is useful for flight training, mission rehearsal, and flight test card development.

A finite element model (FEM) was developed to model the structural vibrations of the aircraft. The FEM includes 25 structural modes tabulated at 17 fuel weights. As the aircraft burns fuel, its mass properties change, which in turn change the structural vibration modes. A ground vibration test (GVT) was conducted and the FEM was tuned to this data [21]. The first four longitudinal vibration mode shapes that were observable in the flight test data discussed later are shown in Figure 2, where modal displacements are exaggerated for clarity. The numbering of the modes corresponds with the FEM ordering. The first longitudinal structural mode is the first symmetric wing bending (SW1B) mode, where the wings and nose of the aircraft deform together in phase while the aft section of the center body bends out of phase. In the first symmetric wing torsion (SW1T) mode, the wings twist together in phase. The symmetric fore-aft (SFA) mode is a longitudinal bending of the wings back and forth, and the symmetric winglet lateral (SWL) mode is a bending of the winglets with additional bending and torsion in the wings. The main structural modes that are of interest to the research effort are the SW1B and SW1T modes.

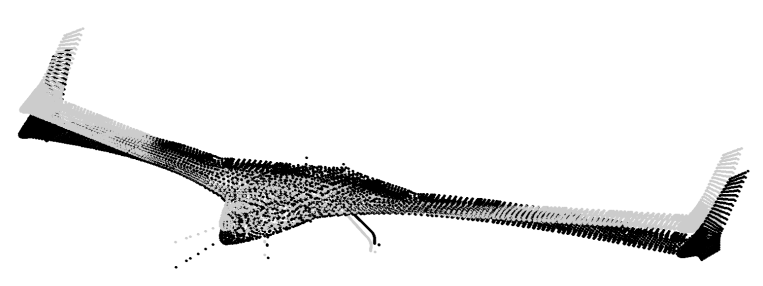

(a)

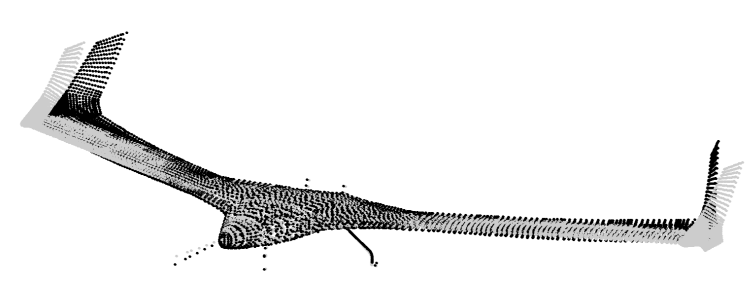

(c)

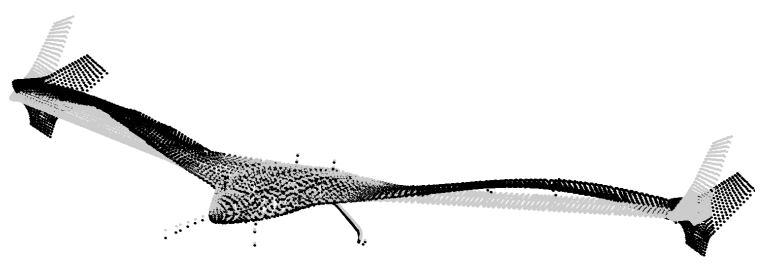

(b)

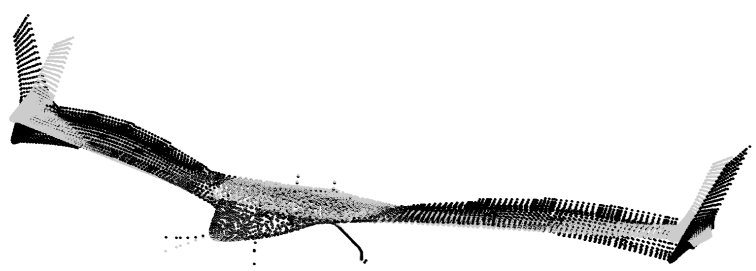

(d)

Figure 2. Longitudinal in-vacuo mode shapes for the X-56A (FEM configuration 24611, version 10.424_dev, 50\% fuel). Gray markers are the undeformed grid points and black markers are the deformed points. (a) Mode 7: First symmetric wing bending (SW1B). (b) Mode 9: First symmetric wing torsion (SW1T). (c) Mode 10: Symmetric fore-aft (SFA). (d) Mode 14: Symmetric winglet lateral (SWL).

The flight test data discussed in Section 6 and the nonlinear simulation indicated that the SW1B mode (the lowest-frequency structural mode) was approximately twice the frequency of the short period mode (the highest-frequency rigid-body mode of interest here). Because this factor was less than five, as recommended in Reference [11], the hybrid-flexible model simplification was not appropriate. Rather, the full Waszak-Schmidt model, containing both the flexible-to-rigid and rigid-to-flexible aeroelastic couplings, was retained for modeling.

\subsection{Flight Test Instrumentation}

The X-56A is equipped with many sensors for studying its aeroelastic characteristics. A planform of the aircraft with naming conventions and locations for relevant sensors and control effectors is shown in Figure 3. 


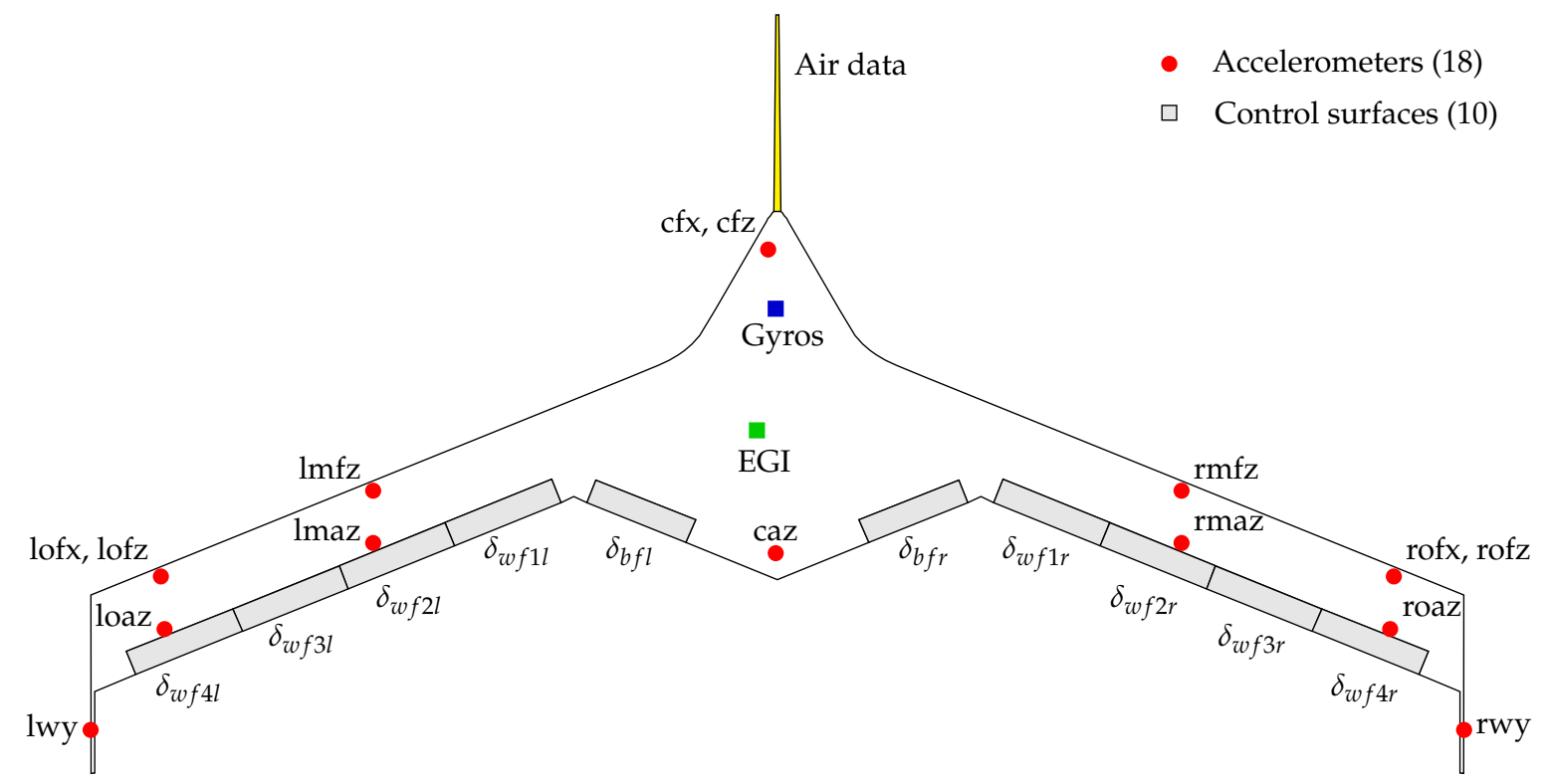

Figure 3. X-56A planform with selected sensor and control surface locations.

The ten trailing-edge control surfaces, shaded gray in Figure 3, are first designated with " $b f$ " for body flap or " $w f$ " for wing flap. The wing flaps are additionally enumerated from the wing root to wing tip. The designations end with " $l$ " or " $r$ " for left or right. All control deflections were measured using string potentiometers and are considered positive with trailing-edge down deflection.

The 18 accelerometers, drawn as red dots in Figure 3, consist of 4 longitudinal, 3 lateral, and 11 vertical sensors. The embedded GPS/INS (EGI) system, drawn as a green square and located near the nominal center of mass, contains three of these accelerometers. The center-body and wing accelerometers are designated first with an " $l$ " for left wing, " $c$ " for center body, or " $r$ " for right wing; then " $w$ " for winglet, " $o$ " for outboard, or " $m$ " for midspan; followed by " $f$ " for forward, or " $a$ " for aft. The last character is " $x$," " $y$," or " $z$ " and designates the aircraft body axis with which the instrument is aligned.

The EGI also provides an attitude solution and measurements of the angular rates from rate gyroscopes. Additional high-rate gyroscopes are installed in the center body, which is drawn as a blue square in Figure 3. Air data vanes on the boom protruding from the aircraft nose, drawn in yellow, measure angle of attack and flank angle. The nose boom also contains pressure ports, which were combined with temperature and pressure sensor readings to compute airspeed. Differential GPS provides position and inertial velocity information, and 24 strain gauges and a fiber-optic strain sensor (FOSS) are installed in the aircraft.

Sensor measurements were sampled at rates ranging 12-62 times the highest frequency of interest, depending on the sensor. Although Morelli and Klein [22] suggested the sampling rate should be at least 25 times the highest frequency of interest, many quantities, such as airspeed, do not respond quickly and therefore do not need the higher sampling rate. Furthermore, the factor of 12 was already several times faster than sampling rates used in typical flight tests with less flexible aircraft. The measured data were passed through anti-aliasing filters, digitized, and synchronized. As small time skews between data channels, for example created by the attitude estimation filter internal to the EGI, can degrade estimation results [22,23], these time skews were estimated and removed from the data, as described in Section 6. This step was found to be critical in the analysis because time delays manifest as increased phase lags at higher frequencies, such as near the structural modes of interest. 


\section{Experiment Design}

The system identification maneuvers consisted of trimming the aircraft in straight and level flight and then applying excitations on the control surfaces. Because there are many control surfaces, a large frequency bandwidth of interest, many test points, and a relatively fast rate of fuel consumption affecting the mass and aeroelastic properties properties of the aircraft, testing using conventional inputs on sequential control surfaces would have been prohibitive. Instead, orthogonal phase-optimized multisine inputs were used to move all the control surfaces at the same time, but in different ways, over a specified frequency band and with prescribed power spectra. The orthogonal phase-optimized multisine inputs (hereafter referred to as multisines) summarized in this section were first presented in References [24,25] and were elaborated upon in Reference [22] and the references therein.

Multisine input design begins with choosing the time duration $T$, which defines the fundamental frequency $1 / T$. Harmonic frequencies $k / T$ for integer values of $k \in K$ are then selected to span the bandwidth of interest. For $n_{u}$ different inputs to be excited at the same time, the harmonics in $K$ are divided among the subsets $K_{1}, K_{2}, \ldots, K_{n_{u}}$. The multisines for each input are then assembled as

$$
u_{j}(t)=\sum_{k \in K_{j}} a_{k} \sin \left(\frac{2 \pi k}{T} t+\phi_{k}\right), \quad j=1,2, \ldots, n_{u}
$$

which are summations of harmonic sinusoids. The power spectra $a_{k}$ can take a uniform distribution to excite all the harmonic frequencies equally, which is the usual case, or can be designed to emphasize or avoid different modal responses. The $a_{k}$ values for each input are also jointly scaled to achieve responses with good signal-to-noise ratios. The phase spectra $\phi_{k}$ for each input are optimized using a simplex method for the minimum relative peak factor (RPF)

$$
\operatorname{RPF}\left(u_{j}\right)=\frac{\max \left(u_{j}\right)-\min \left(u_{j}\right)}{2 \sqrt{2} \operatorname{rms}\left(u_{j}\right)}
$$

resulting in inputs that generate small perturbation responses about the flight condition, appropriate for linear modeling. Because the sinusoids used in Equation (1) are harmonic multiples, they are mutually orthogonal; therefore, the multisines can be used on multiple control surfaces at the same time without correlating the input data. This property allows flight test time to be considerably reduced by exciting multiple control effectors at the same time instead of in sequence. The multisines are added to other inputs from the pilot and control system just before the actuators. A feedback control law will raise the level of correlation between the inputs, but when configured in this manner, input pairwise correlations remain well below the 0.9 guideline suggested in Reference [22] and facilitate accurate estimation of the bare-airframe dynamics using the output-error approach. The actual model structure to be identified is not important at this point (beyond that the relevant dynamics are well excited); rather, it is only important that the inputs are sufficiently different so that the responses resulting from each input can be distinguished.

For the X-56A Phase 1 flight tests, 72 different multisine inputs were designed for identification of the bare-airframe dynamics using different combinations of control surfaces and engine throttles for various amplitudes and frequency ranges. For the excitation discussed in this paper (flight test aid (FTA) 506), the control surfaces on the left and right wings were moved symmetrically. For example, the symmetric body flap deflections were defined as

$$
\delta_{b f s}=\frac{1}{2}\left(\delta_{b f l}+\delta_{b f r}\right)
$$

where the "s" subscript stands for symmetric. This setup resulted in five multisines for the symmetric control surface pairs. The time duration $T$ was chosen to produce a short maneuver with adequate frequency resolution over the modal responses of interest for five inputs. The adequacy of the frequency 
resolution was confirmed prior to flight testing using the nonlinear simulation, and the estimated changes in mass properties were judged to be sufficiently small for linear modeling. The bandwidth of the included harmonic frequencies spanned short period (SP) mode and the four vibration modes shown in Figure 2. In total, 325 harmonic frequencies were used, with 65 frequencies on each input. The ordering of the frequencies among the five inputs was: $\delta_{b f s}, \delta_{w f 3 s}, \delta_{w f 1 s}, \delta_{w f 4 s}$, and $\delta_{w f 2 s}$, which ensured that sequential frequencies were distributed over the wing and not excited on neighboring control surfaces. The power spectra for these multisines were uniform in frequency, and the amplitudes were adjusted using a nonlinear simulation of the aircraft to attain good expected signal-to-noise ratios under a variety of conditions.

Measurements of control surfaces deflections are shown in Figure 3a. The maneuver contained approximately 2.5 cycles of the input, but only one cycle is shown for clarity. The y-axes have the same sizes but different mean values for each input. These measurements include not only the multisine perturbation inputs, but also contributions from the pilot and control system. In the first part of the maneuver before the excitation onset, the aircraft is essentially trimmed and the relatively low-frequency movement of the control surfaces is from the control system. The multisines were designed to be orthogonal but the control mixing and feedback raised the maximum control surface correlation to 0.13 . This correlation was well below the 0.9 guideline and was not an issue during the identification analysis.

Fourier transforms of the measured control surface data (computed using the method discussed later in Section 5) are shown as amplitude spectra in Figure 3b. The transforms were only evaluated at the harmonic frequencies used in the multisine inputs. This choice restricted the number of frequency-domain data points to those having the input power, thereby reducing the computational complexity of the analysis without reducing the amount of information in the modeling data. Modeling results did not significantly improve with more frequency points. The ranges for the axes in each plot again have the same scale. Resonant frequencies for the short period and in-vacuo structural modes are annotated in Figure $3 \mathrm{~b}$. The actuator bandwidth created some amplitude attenuation with increasing frequency. Although the inputs were designed to have unique frequencies for each control surface, control mixing and feedback control changed the frequency content near the short period and SW1B modes for $\delta_{b f s}, \delta_{w f 2 s}, \delta_{w f 3 s}$, and $\delta_{w f 4 s}$. 


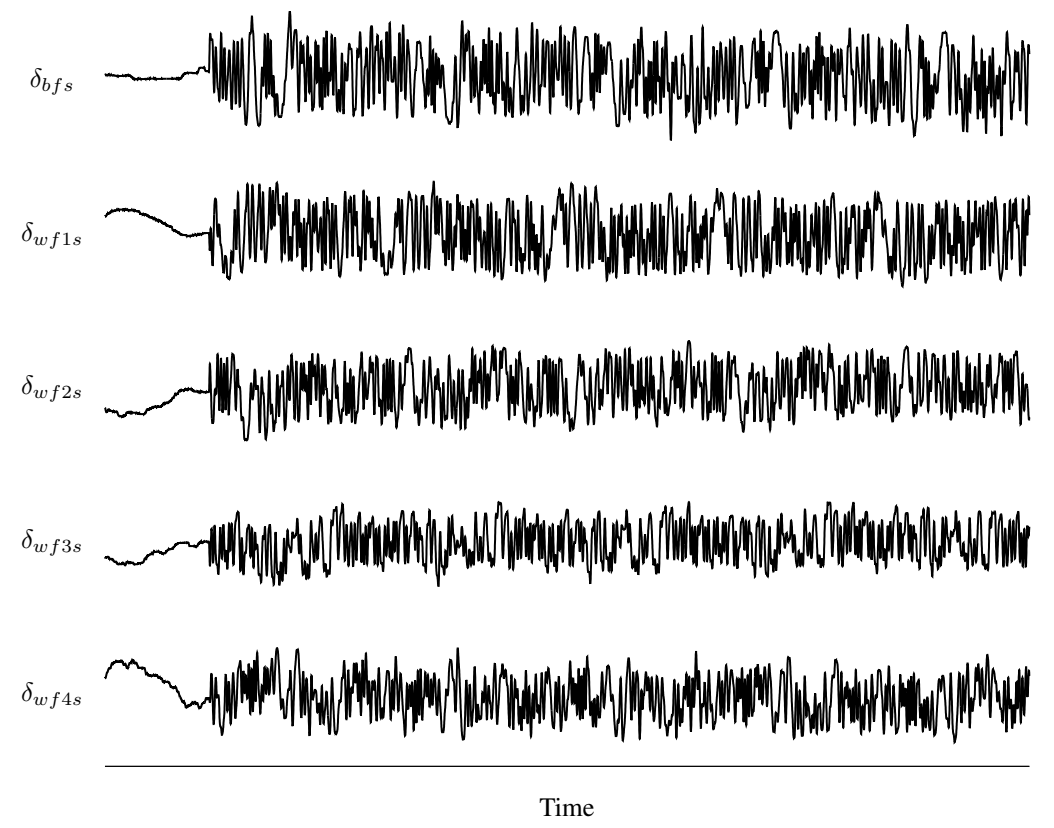

(a)
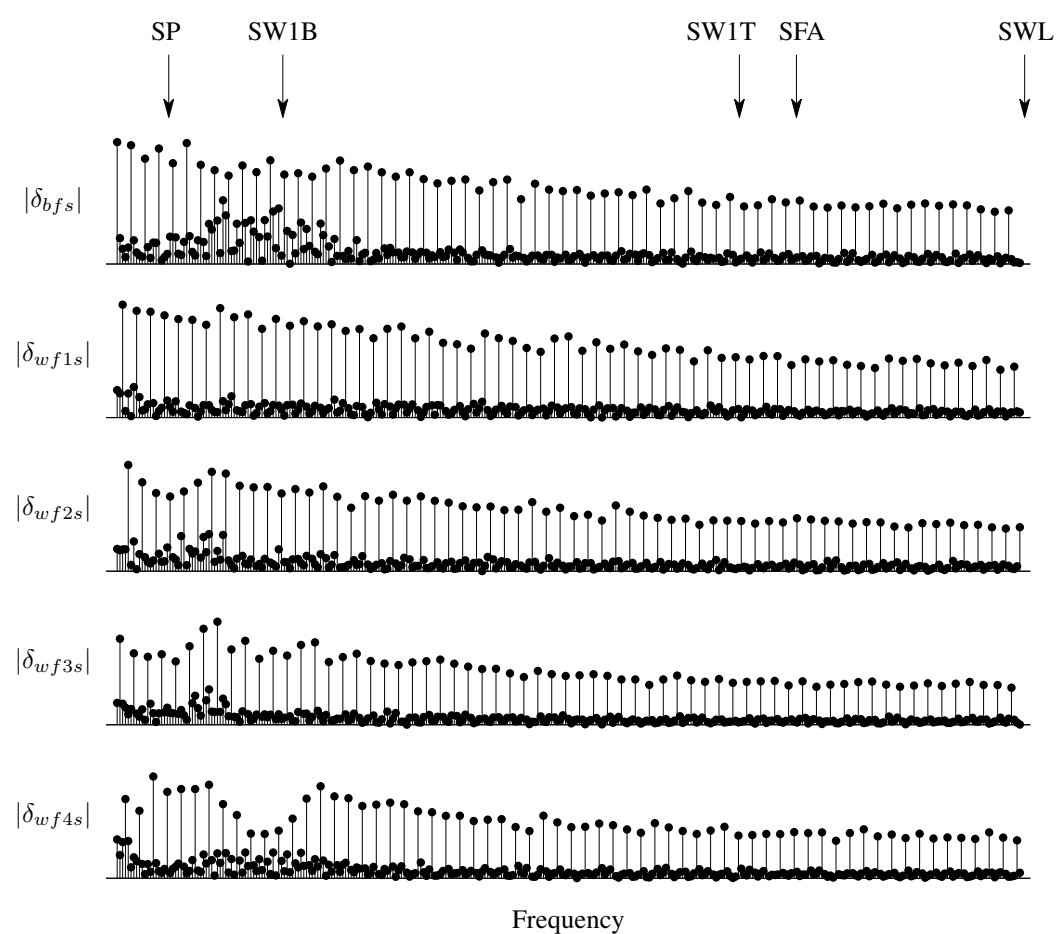

(b)

Figure 3. Control surface deflections (Flight 18, FTA 506, 32\% fuel, 80\% flutter speed): (a) measured time histories; and (b) Fourier transform amplitude spectra with approximate modal frequencies noted. 


\section{Aeroelastic Flight Dynamics Model}

This section presents the aeroelastic flight dynamics model used for identification, which is based on References [3,5]. Sections 4.1 and 4.2 summarize the structural dynamics and how those motions couple into the aeroelastic forces and moments. Linearized equations of motion in state-space format are introduced in Section 4.3. Linearized output equations, developed in Reference [26], are given in Section 4.4.

\subsection{Structural Dynamics}

The aircraft structure is assumed to undergo small displacements within the linear elastic range. The displacement of an arbitrary point on the aircraft

$$
\mathbf{d}(\mathbf{r}, t)=\sum_{k=1}^{n_{m}} \boldsymbol{\phi}_{k}(\mathbf{r}) \eta_{k}(t)
$$

arising from this structural deformation is parameterized in terms of orthogonal, in-vacuo vibration modes that are separable in space and time. The vector $\phi_{k}$ is the mode shape of the $k$ th vibration mode and quantifies the linear displacement of the structure at the point $\mathbf{r}$. The scalar $\eta_{k}$ is the generalized modal displacement of the $k$ th mode and quantifies the participation of the mode in time. Although in theory an infinite number of structural modes exist, practical constraints and data information limitations restrict the model to a small, finite number of $n_{m}$ modes.

Each vibration mode was modeled as the second-order system

$$
\ddot{\eta}_{i}+2 \zeta_{i} \omega_{i} \dot{\eta}_{i}+\omega_{i}^{2} \Delta \eta_{i}=\frac{\bar{q} S \bar{c}}{m_{i}} C_{Q_{i}} \quad i=1,2, \ldots, n_{m}
$$

about the local equilibrium, where $m_{i}$ is the modal mass, $\omega_{i}$ is the modal frequency, and $\zeta_{i}$ is the modal damping ratio. The non-dimensional generalized force coefficient $C_{Q_{i}}$ quantifies the aerodynamic forcing of the structural mode and is developed next in Section 4.2 using stability and control derivatives. The parameters $m_{i}, \omega_{i}, \zeta_{i}$, and $\phi_{i}$ are usually obtained from a FEM analysis and a GVT, and vary with the mass properties of a given aircraft, for example due to fuel burn.

It is important to note that this model of the structural modes describes the in-vacuo vibration of the aircraft when air is not present. These modes are be used as basis functions to describe the motion of the aircraft structure when air is present. Structural deformations can be parameterized using any basis set, such as in Equation (4), but this particular choice has physical significance and uses parameters that can be modeled or measured using standard techniques. Moreover, choosing the in-vacuo structural modes as basis functions has a theoretical justification and significantly reduces the size of databases for parameterizing the structural dynamics at different conditions.

\subsection{Aeroelastic Forces and Moments}

The aeroelastic forces and moments acting on the aircraft were modeled using non-dimensional stability and control derivatives in truncated first-order Taylor series expansions. The longitudinal force and moment coefficient perturbations were

$$
\begin{aligned}
& \Delta C_{Z}=C_{Z_{\alpha}} \Delta \alpha+C_{Z_{q}} \frac{q \bar{c}}{2 V}+\sum_{k=1}^{n_{m}} C_{Z_{\eta_{k}}} \Delta \eta_{k}+\sum_{k=1}^{n_{m}} C_{Z_{\dot{\eta}_{k}}} \frac{\dot{\eta}_{k} \bar{c}}{2 V}+C_{Z_{\delta}} \Delta \delta \\
& \Delta C_{m}=C_{m_{\alpha}} \Delta \alpha+C_{m_{q}} \frac{q \bar{c}}{2 V}+\sum_{k=1}^{n_{m}} C_{m_{\eta_{k}}} \Delta \eta_{k}+\sum_{k=1}^{n_{m}} C_{m_{\eta_{k}}} \frac{\dot{\eta}_{k} \bar{c}}{2 V}+C_{m_{\delta}} \Delta \delta
\end{aligned}
$$

which involved the short period mode and the relevant structural modes. These equations are formed in the body mean-axes and are valid for small perturbations about the equilibrium condition. Due 
to the flexibility of the aircraft and the mean-axis assumptions, other reference frames, such as the stability axes, should not be used for modeling. The first, second, and last terms on the right sides of Equation $(6 a, b)$ are used in models of traditional rigid-body aircraft. The third and fourth terms, which are unique to models of flexible aircraft, quantify the effect of the structural motion of the aircraft on the aerodynamic vertical force and pitching moment.

Similarly, the non-dimensional generalized force coefficient in Equation (5) was modeled as

$$
\Delta C_{Q_{i}}=C_{Q_{i_{\alpha}}} \Delta \alpha+C_{Q_{i q}} \frac{q \bar{c}}{2 V}+\sum_{k=1}^{n_{m}} C_{Q_{i_{\eta_{k}}}} \Delta \eta_{k}+\sum_{k=1}^{n_{m}} C_{Q_{i_{\eta_{k}}}} \frac{\dot{\eta}_{k} \bar{c}}{2 V}+C_{Q_{i_{\delta}}} \Delta \delta, \quad i=1,2, \ldots, n_{m}
$$

Each of the stability and control derivatives in Equation (7) describes the forcing of the corresponding structural mode arising from perturbations in the states or controls. For example, $C_{Q_{7}}$ quantifies the symmetric bending mode force resulting from a control surface displacement. The off-axis derivatives, for example $C_{Z_{\eta_{k}}}$ or $C_{Q_{i_{\alpha}}}$, quantify the aeroelastic interactions between the traditional aircraft flight dynamics and the structural motion of the aircraft.

\subsection{State Equations}

Given the structural model developed in Section 4.1 and the aeroelastic forces and moments defined in Section 4.2, the full aircraft equations of motion can be linearized about a reference condition and cast into the canonical state-space format

$$
\dot{\mathbf{x}}=\mathbf{A x}+\mathbf{B u}
$$

The state vector $\mathbf{x}$ in Equation (8) can be partitioned as

$$
\mathbf{x}=\left[\begin{array}{l}
\mathbf{x}_{r} \\
\mathbf{x}_{e}
\end{array}\right]
$$

where $\mathbf{x}_{r}$ are states of the mean axes and are traditionally associated with rigid-body aircraft, and where $\mathbf{x}_{e}$ are the modal displacements and rates associated with the elastic vibration of the structure. For the model identified below, the partitioned states were

$$
\mathbf{x}_{r}=\left[\begin{array}{c}
\Delta \alpha \\
q \\
\Delta \theta
\end{array}\right], \quad \mathbf{x}_{e}=\left[\begin{array}{c}
\Delta \eta_{7} \\
\Delta \eta_{9} \\
\dot{\eta}_{7} \\
\dot{\eta}_{9}
\end{array}\right]
$$

The mean-axis states include the angle of attack, pitch rate, and pitch angle, whereas the modal states are the displacements and rates of the SW1B and SW1T modes. The SFA and SWL modes, while present in the flight test data examined below, did not significantly impact the system identification analysis in this paper or in References [17,18], and were therefore neglected. The inputs to the model were perturbations in the symmetric control surface pairs

$$
\mathbf{u}=\left[\begin{array}{c}
\Delta \delta_{b f s} \\
\Delta \delta_{w f 1 s} \\
\Delta \delta_{w f 2 s} \\
\Delta \delta_{w f 3 s} \\
\Delta \delta_{w f 4 s}
\end{array}\right]
$$


Using these definitions, Equation (8) can be partitioned as

$$
\left[\begin{array}{c}
\dot{\mathbf{x}}_{r} \\
\dot{\mathbf{x}}_{e}
\end{array}\right]=\left[\begin{array}{cc}
\mathbf{A}_{r r} & \mathbf{A}_{r e} \\
\mathbf{A}_{e r} & \mathbf{A}_{e e}
\end{array}\right]\left[\begin{array}{c}
\mathbf{x}_{r} \\
\mathbf{x}_{e}
\end{array}\right]+\left[\begin{array}{c}
\mathbf{B}_{r} \\
\mathbf{B}_{e}
\end{array}\right] \mathbf{u}
$$

First examining the A matrix, the partitioned submatrices were

$$
\begin{aligned}
& \mathbf{A}_{r r}=\left[\begin{array}{ccc}
Z_{\alpha}-X_{T} \cos \alpha / m V & 1+Z_{q} & 0 \\
M_{\alpha} & M_{q} & 0 \\
0 & 1 & 0
\end{array}\right] \\
& \mathbf{A}_{r e}=\left[\begin{array}{cccc}
Z_{\eta_{7}} & Z_{\eta_{9}} & Z_{\dot{\eta}_{7}} & Z_{\dot{\eta}_{9}} \\
M_{\eta_{7}} & M_{\eta_{9}} & M_{\dot{\eta}_{7}} & M_{\dot{\eta}_{9}} \\
0 & 0 & 0 & 0
\end{array}\right] \\
& \mathbf{A}_{e r}=\left[\begin{array}{ccc}
0 & 0 & 0 \\
0 & 0 & 0 \\
Q_{7_{\alpha}} & Q_{7_{q}} & 0 \\
Q_{9_{\alpha}} & Q_{9_{q}} & 0
\end{array}\right] \\
& \mathbf{A}_{e e}=\left[\begin{array}{cccc}
0 & 0 & 0 & 0 \\
0 & 0 & 0 & 0 \\
Q_{7_{\eta_{7}}} & Q_{7_{\eta_{9}}} & Q_{7_{\eta_{7}}} & Q_{7_{\eta_{9}}} \\
Q_{\eta_{\eta_{7}}} & Q_{9_{\eta_{9}}} & Q_{9_{\eta_{7}}} & Q_{9_{\eta_{9}}}
\end{array}\right]+\left[\begin{array}{cccc}
0 & 0 & 1 & 0 \\
0 & 0 & 0 & 1 \\
-\omega_{7}^{2} & 0 & -2 \zeta_{7} \omega_{7} & 0 \\
0 & -\omega_{9}^{2} & 0 & -2 \zeta_{9} \omega_{9}
\end{array}\right]
\end{aligned}
$$

For notational convenience, the submatrices in Equation (13) have been expressed in terms of dimensional stability and control derivatives, defined in terms of the non-dimensional derivatives as

$$
\begin{array}{rllll}
Z_{\alpha}=\frac{\bar{q} S}{m V} C_{Z_{\alpha}} & Z_{q}=\frac{\bar{q} S \bar{c}}{2 m V^{2}} C_{Z_{q}} & Z_{\eta_{k}}=\frac{\bar{q} S}{m V} C_{Z_{\eta_{k}}} & Z_{\dot{\eta}_{k}}=\frac{\bar{q} S \bar{c}}{2 m V^{2}} C_{Z_{\eta_{\eta_{k}}}} & Z_{\delta}=\frac{\bar{q} S}{m V} C_{Z_{\delta}} \\
M_{\alpha}=\frac{\bar{q} S \bar{c}}{I_{y y}} C_{m_{\alpha}} & M_{q}=\frac{\bar{q} S \bar{c}^{2}}{2 V I_{y y}} C_{m_{q}} & M_{\eta_{k}}=\frac{\bar{q} S \bar{c}}{I_{y y}} C_{m_{\eta_{k}}} & M_{\dot{\eta}_{k}}=\frac{\bar{q} S \bar{c}^{2}}{2 V I_{y y}} C_{m_{\eta_{k}}} & M_{\delta}=\frac{\bar{q} S \bar{c}}{I_{y y}} C_{m_{\delta}} \\
Q_{i_{\alpha}}=\frac{\bar{q} S \bar{c}}{m_{i}} C_{Q_{i_{\alpha}}} & Q_{i_{q}}=\frac{\bar{q} S \bar{c}}{2 m_{i} V^{2}} C_{Q_{i_{q}}} & Q_{i_{\eta_{k}}}=\frac{\bar{q} S \bar{c}}{m_{i}} C_{Q_{i_{\eta_{k}}}} & Q_{i_{\eta_{k}}}=\frac{\bar{q} S \bar{c}}{2 m_{i} V^{2}} C_{Q_{i_{\eta_{k}}}} & Q_{i_{\delta}}=\frac{\bar{q} S \bar{c}}{m_{i}} C_{Q_{i_{\delta}}}
\end{array}
$$

where $X_{T}$ is the thrust force aligned with the longitudinal body axis. The submatrix $\mathbf{A}_{r r}$ describes forces and moments imparted on the mean-axis states by the mean-axis states, and is a traditional model for the short-period approximation of rigid-body aircraft at low angles of attack. The submatrix $\mathbf{A}_{e e}$ describes the generalized forces imparted on the modal states by the modal states and is typical of vibration models. In Equation (13d), the submatrix has been further partitioned into contributions arising from the generalized aerodynamic forces (first term) and from the structural stiffness and damping (second term). The submatrices $\mathbf{A}_{r e}$ and $\mathbf{A}_{e r}$ represent aeroelastic couplings between the mean-axis and modal states that arise through aerodynamic interactions. In the hybrid-flexible model [10-12] mentioned above, it is the $\mathbf{A}_{r e}$ matrix that is neglected by setting it equal to zero.

Next, looking at the $\mathbf{B}$ matrix, the submatrices were

$$
\mathbf{B}_{r}=\left[\begin{array}{ccccc}
Z_{\delta_{b f s}} & Z_{\delta_{w f 1 s}} & Z_{\delta_{w f 2 s}} & Z_{\delta_{w f 3 s}} & Z_{\delta_{w f 4 s}} \\
M_{\delta_{b f s}} & M_{\delta_{w f 1 s}} & M_{\delta_{w f 2 s}} & M_{\delta_{w f 33 s}} & M_{\delta_{w f 4 s}} \\
0 & 0 & 0 & 0 & 0
\end{array}\right]
$$




$$
\mathbf{B}_{e}=\left[\begin{array}{ccccc}
0 & 0 & 0 & 0 & 0 \\
0 & 0 & 0 & 0 & 0 \\
Q_{\gamma_{\delta_{b f s}}} & Q_{7_{\delta_{w f 1 s}}} & Q_{7_{\delta_{w f 2 s}}} & Q_{7_{\delta_{w f 3 s}}} & Q_{7_{\delta_{w f 4 s}}} \\
Q_{9_{\delta_{b f s}}} & Q_{9_{\delta_{w f 1 s}}} & Q_{\delta_{\delta_{w f 2 s}}} & Q_{9_{\delta_{w f 3 s}}} & Q_{9_{\delta_{w f 4 s}}}
\end{array}\right]
$$

The matrix $\mathbf{B}_{r}$ describes the forces and moments developed on the mean-axis states by the control deflections, and the matrix $\mathbf{B}_{e}$ describes the effects of the control surface deflections on the modal states.

\subsection{Output Equations}

The output equations for pertinent sensor measurements described in this section are summarized from Reference [26], in which both nonlinear and linear output equations were developed for flexible aircraft models. The linearized output responses were cast into the state-space format

$$
\mathbf{y}=\mathbf{C x}+\mathbf{D u}
$$

The air flow angle vane mounted on the X-56A nose boom measures the local angle of attack at the rotational axis of the vane. The linearized equation for this measurement is

$$
\alpha^{v n}=\alpha+\frac{x_{v n}}{V} q+\sum_{k=1}^{n_{m}} \frac{\phi_{k_{z}}^{v n}}{V} \dot{\eta}_{k}
$$

The first term on the right side of Equation (17) is the angle of attack state at the aircraft center of mass, and the second term is the angular rate contribution from the sensor offset of $x_{v n}$ along the $x$-body axis. The third term describes contributions from the modal rates at the sensor. The quantity $\phi_{k_{z}}^{v n}$ is the linear displacement of the sensor along the $z$-body axis arising from a unit displacement of $\eta_{k}$.

The high-rate gyroscope installed near the X-56A nose measures the local angular rate at the sensor

$$
q^{g y r}=q+\sum_{k=1}^{n_{m}} v_{k_{\theta}}^{g y r} \dot{\eta}_{k}
$$

The first term on the right side of Equation (18) is the pitch rate of the mean axes. The second term contains the local contributions of the aircraft structural deformation. The term $v_{k_{\theta}}^{g y r}$ quantifies the angular pitch displacement of the sensor from a unit displacement of $\eta_{k}$, analogous to the linear displacements described by $\boldsymbol{\phi}_{k}$. Similarly, measurements of the Euler angles from the EGI

$$
\theta^{e g i}=\theta+\sum_{k=1}^{n_{m}} v_{k_{\theta}}^{e g i} \eta_{k}
$$

contain contributions from the mean-axis pitch angle and the local angular deformation of the aircraft structure.

Vertical accelerometers measure translational acceleration due to applied forces, excluding gravity. In general, these measurements are complicated expressions involving the forces, angular rates and accelerations, and the modal displacements, rates, and accelerations. In the linearized case when using the aerodynamic forces in Equation (6a), the output equation for longitudinal flight using the included states and controls simplifies to

$$
a_{z}^{a c c}=\frac{\bar{q} S}{m g} \Delta C_{Z}-\frac{x_{a c c}}{g} \dot{q}+\sum_{k=1}^{n_{m}} \frac{\phi_{k_{z}}^{a c c}}{g} \ddot{\eta}_{k}
$$


where the generic label "acc" can stand for any of the sensor location names. The first two terms on the right side of Equation (20) are due to the applied aerodynamic forces and position offsets from the mass center. The last term contains the local acceleration at the sensor due to structural deformation.

These output equations for different sensors are used to populate the state-space model in Equation (16). As discussed in Section 6, these measurements are also subject to errors including measurement noise, bias and scale factor errors, and relative time skews. Transducer dynamics for the sensors, obtained from ground testing, and the effects of digital filters were negligible over the frequency range of interest in the flight data discussed.

\section{Output-Error Parameter Estimation in the Frequency Domain}

The output-error approach for parameter estimation is the maximum likelihood estimator for dynamic systems where process noise (for example, turbulence) is neglected. A set of unknown model parameters are determined that best match the model outputs to measured output data. For this analysis, the outputs were Fourier transforms of the aircraft responses and the model parameters were non-dimensional stability and control derivatives in the Waszak-Schmidt model. A brief summary of the output-error approach using frequency-domain data, based on References [22,27], is presented in this section.

To perform the estimation in the frequency domain, the finite Fourier transform

$$
x(j \omega)=\int_{0}^{T} x(t) e^{-j \omega t} d t
$$

is used to convert detrended time-domain data into frequency-domain data, where $T$ is the time duration of the data record of $x(t)$ and where $\omega$ is the radian frequency. Fast Fourier transforms (FFTs) could be used to implement the Fourier transform, but these have relatively coarse frequency resolution for aircraft flight dynamics applications. Rather, Equation (21) can be computed to arbitrary resolution and a high degree of accuracy using the chirp z-transform and cubic interpolation of the time series data [22,28]. Because the Fourier transform is a linear operator, dynamic models used in frequency-domain output error are restricted to linear systems. However, this is a mild restriction in this case because the flight test data involved small-perturbations about a reference flight condition and were represented well using linearized models.

Applying the Fourier transform to the state-space model assembled from Equations (8) and (16) yielded the input-output relation

$$
\mathbf{y}(j \omega)=\left[\mathbf{C}(j \omega \mathbb{I}-\mathbf{A})^{-1} \mathbf{B}+\mathbf{D}\right] \mathbf{u}(j \omega)
$$

in the frequency domain assuming zero initial conditions. The measured output was modeled as

$$
\mathbf{z}(j \omega)=\mathbf{y}(j \omega)+\mathbf{v}(j \omega)
$$

where the additive measurement noise $\mathbf{v}$ had

$$
\begin{aligned}
E[\mathbf{v}(j \omega)] & =\mathbf{0} \\
E\left[\mathbf{v}(j \omega) \mathbf{v}(j \omega)^{\dagger}\right] & =\frac{1}{N} \mathbf{S}_{v v}
\end{aligned}
$$

where $\mathbf{S}_{v v}$ is the spectral density of $\mathbf{v}(t)$ and where $N$ is the number of frequencies analyzed.

The maximum likelihood cost function to be minimized for the unknown model parameters in the output-error approach with frequency-domain data is

$$
J(\boldsymbol{\theta})=N \sum_{k=1}^{N} \boldsymbol{v}\left(j \omega_{k}\right)^{\dagger} \mathbf{S}_{v v}^{-1} \boldsymbol{v}\left(j \omega_{k}\right)+N \ln \left|\mathbf{S}_{v v}\right|
$$


where $\theta$ is the vector of unknown model parameters and the residuals are

$$
\boldsymbol{v}\left(j \omega_{k}\right)=\mathbf{z}\left(j \omega_{k}\right)-\mathbf{y}\left(j \omega_{k}\right)
$$

In the past, attempts to minimize the cost function in Equation (25) for both $\boldsymbol{\theta}$ and $\mathbf{S}_{v v}$ at the same time have led to convergence problems. To overcome this, a relaxation technique is typically employed to alternate solving between one of these two unknowns while holding the other constant. This technique has been shown to work well in practice.

For a given $\boldsymbol{\theta}$, minimizing the cost function for the residual spectral density matrix leads to

$$
\hat{\mathbf{S}}_{v v}=\sum_{k=1}^{N} \boldsymbol{v}\left(j \omega_{k}\right) \boldsymbol{v}\left(j \omega_{k}\right)^{\dagger}
$$

While holding this matrix constant, Equation (25) can then be minimized for the model parameters as

$$
J(\boldsymbol{\theta})=N \sum_{k=1}^{N} \boldsymbol{v}\left(j \omega_{k}\right)^{\dagger} \hat{\mathbf{S}}_{v v}^{-1} \boldsymbol{v}\left(j \omega_{k}\right)
$$

Optimization of Equation (28) can be done iteratively from starting values for the model parameters using the Gauss-Newton method. This technique is similar to the Newton-Raphson method but does not require the Hessian matrix to be computed. The iteration update for the model parameters is

$$
\hat{\boldsymbol{\theta}}=\hat{\boldsymbol{\theta}}_{0}-\mathbf{M}_{\boldsymbol{\theta}=\hat{\boldsymbol{\theta}}_{0}}^{-1}\left[\frac{\partial J(\boldsymbol{\theta})}{\partial \boldsymbol{\theta}}\right]_{\boldsymbol{\theta}=\hat{\boldsymbol{\theta}}_{0}}
$$

The matrix

$$
\mathbf{M}=E\left[\frac{\partial^{2} J(\boldsymbol{\theta})}{\partial \boldsymbol{\theta} \partial \boldsymbol{\theta}^{T}}\right]=2 N \Re\left[\sum_{k=1}^{N} \mathbf{S}^{\dagger}\left(j \omega_{k}\right) \hat{\mathbf{S}}_{v v}^{-1} \mathbf{S}\left(j \omega_{k}\right)\right]
$$

is the Fisher information matrix, where

$$
\mathbf{S}\left(j \omega_{k}\right)=\frac{\partial \mathbf{y}\left(j \omega_{k}, \boldsymbol{\theta}\right)}{\partial \boldsymbol{\theta}}
$$

is the output sensitivity matrix, which can be computed analytically by taking derivatives of Equation (22) as

$$
\frac{\partial \mathbf{y}\left(j \omega_{k}, \boldsymbol{\theta}\right)}{\partial \boldsymbol{\theta}}=\mathbf{C}\left(j \omega_{k} \mathbb{I}-\mathbf{A}\right)^{-1}\left[\frac{\partial \mathbf{A}}{\partial \boldsymbol{\theta}} \mathbf{x}\left(j \omega_{k}\right)+\frac{\partial \mathbf{B}}{\partial \boldsymbol{\theta}} \mathbf{u}\left(j \omega_{k}\right)\right]+\frac{\partial \mathbf{C}}{\partial \boldsymbol{\theta}} \mathbf{x}\left(j \omega_{k}\right)+\frac{\partial \mathbf{D}}{\partial \boldsymbol{\theta}} \mathbf{u}\left(j \omega_{k}\right)
$$

or numerically using finite differences. If the sensitivities are computed using the analytical derivatives, the state solution used in Equation (32) is

$$
\mathbf{x}(j \omega)=(j \omega \mathbb{I}-\mathbf{A})^{-1} \mathbf{B} \mathbf{u}(j \omega)
$$

similar to Equation (22). The optimization runs faster using analytical derivatives rather than numerical derivatives because obtaining finite differences requires perturbing each of the many unknown model parameters and computing the outputs. In Equation (29), the matrix

$$
\frac{\partial J(\boldsymbol{\theta})}{\partial \boldsymbol{\theta}}=-2 N \Re\left[\sum_{k=1}^{N} \mathbf{S}\left(j \omega_{k}\right)^{+} \hat{\mathbf{S}}_{v v}^{-1} \boldsymbol{v}\left(j \omega_{k}\right)\right]
$$

is the cost function gradient. The uncertainty in the estimated parameters is given by the Cramér-Rao bounds

$$
\operatorname{cov}[\hat{\boldsymbol{\theta}}]=\mathbf{M}^{-1}
$$


where the square root of the diagonal elements are the standard errors.

Of the many techniques for parameter estimation, output error has many benefits. First, it has a strong theoretical underpinning based in maximum likelihood, and has been used extensively for decades. The assumptions used in output error are physically relevant and reflect flight testing in calm weather. The analysis can incorporate many sensors and can be used with states that are not directly observable, such as the modal displacements and rates. Drawbacks of using output error include that starting values are needed for the unknown model parameters, iterations are required for the nonlinear optimization, and additional effort is needed to change the structure of the model.

Performing the modeling using frequency-domain data also has many benefits. First, viewing the data in the frequency domain provides insight into the modal resonances of the system, such as the structural modes. Second, the computational analysis runs faster in the frequency domain than the time domain, especially when analytically-derived sensitivity equations are used. One reason for this is that the equations of motion are solved in the frequency domain using addition and multiplication, per Equation (22), rather than in the time domain using convolution. Another reason is that there are typically fewer data points to process in the frequency domain than in the time domain. A third reason is that because bias and trend information is removed from the data before applying Fourier transforms, biases in the state and output equations are not estimated. These factors reduce the total number of parameters to be estimated and lessens the computation time required for each optimization iteration. Lastly, analysis with output error in the frequency domain does not require any corrections to the estimated uncertainty levels, which is usually done with time-domain data to correct for colored residuals not assumed by the theory. The frequency-domain output-error analysis is restricted to linear systems, but this was not a drawback for the results presented in this paper because the maneuvers involved small perturbations about a reference condition.

\section{Results}

Measured outputs for the identification maneuver are shown in Figure 4. The inputs for this maneuver are shown in Figure 3 over the same time duration. Many other output measurements were available but were not used in this analysis to keep the parameter estimation problem smaller. In addition, measurements from accelerometers on the left and right wings were averaged to reduce the number of outputs, for example as

$$
a_{z}^{s m f}=\frac{1}{2}\left(a_{z}^{l m f}+a_{z}^{r m f}\right)
$$

similar to the control surface deflections. At the start of the data record, the aircraft was approximately in straight and level flight. There was some motion of the aircraft due to setting up the flight condition, dynamic coupling of the system, and light turbulence. The variations in the response measurements after the onset of the multisine excitations were small and not much bigger than before the onset, but nonetheless produced good data for modeling due to the high-quality sensors used. Spectral analysis showed that the apparent high levels of noise in the accelerometers installed in the center body and wing (for example, $a_{z}^{\text {soa }}$ ) relative to $a_{z}^{\text {egi }}$ were instead due to the presence of many high-frequency structural modes in the data. Variations in the other flight variables were very small, for example a $1.2 \%$ variation in airspeed, $0.3 \%$ change in altitude, $0.4 \%$ change in mass resulting from a $5.5 \%$ decrease in fuel, and $0.1 \%$ change in pitching moment of inertia. The average fuel weight condition for this maneuver was $32 \%$ and the average airspeed was about $80 \%$ of the flutter speed. 


$$
\alpha^{v n}
$$

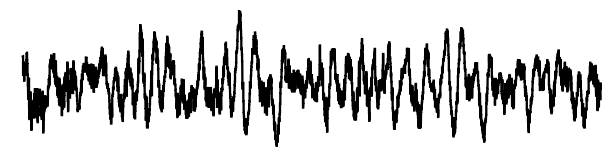

$\theta^{e g i}$

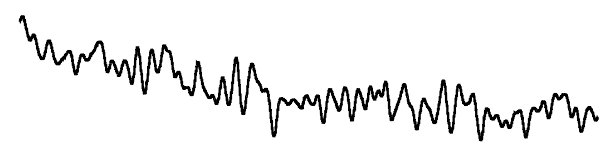

$a_{z}^{c f}$

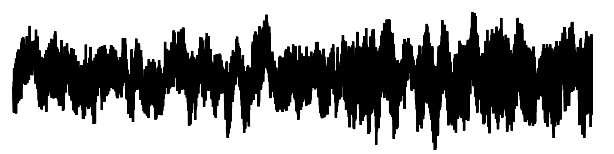

$$
a_{z}^{s m f}
$$

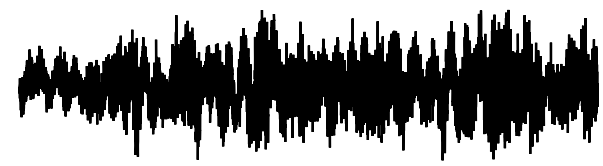

$$
a_{z}^{\text {sof }}
$$

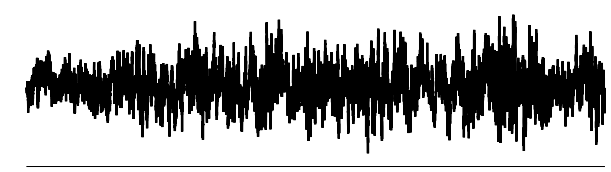

Time
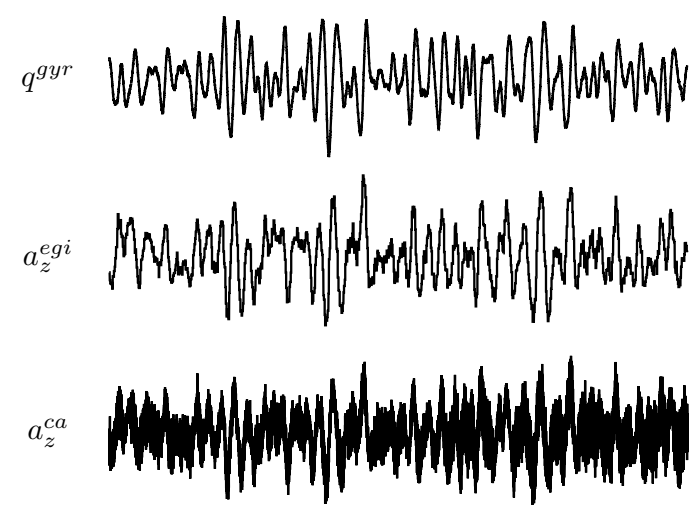

$a_{z}^{s m a}$
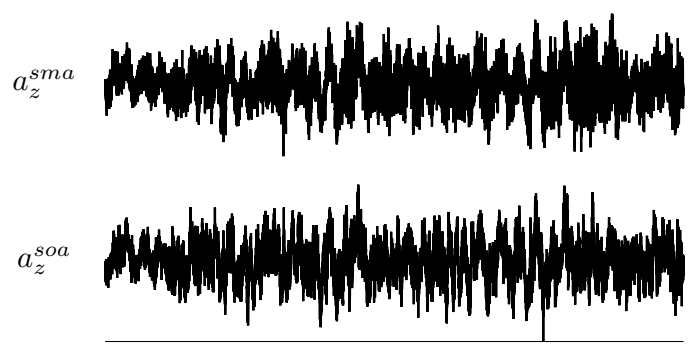

Time

Figure 4. Measured output responses (Flight 18, FTA 506, 32\% fuel, 80\% flutter speed).

Measured input and output data were transformed into the frequency domain at the harmonic frequencies in the multisine inputs. Using only these frequencies in the transforms reduced the computational complexity for the optimization. Estimation results did not significantly change when additional frequencies were used since the input power was concentrated in these frequencies; however, the amount of computation time needed to run the output-error analysis substantially increased.

Simple methods were used to estimate scale factor errors in the angle of attack and pitch angle data, as well as time skews for all measurement Fourier transforms relative to the accelerometer data $[11,29]$. The Fourier transform data were then corrected for these errors. Because there is no kinematic redundancy between the control surface deflections and output responses, a delay for the input $\tau_{\delta}$ was added to Equation (22) as

$$
\mathbf{y}(j \omega)=\left[\mathbf{C}(j \omega \mathbb{I}-\mathbf{A})^{-1} \mathbf{B}+\mathbf{D}\right] \mathbf{u}(j \omega) e^{-j \omega \tau_{\delta}}
$$

to account for the time skew between the input and output measurement data buses. The input time skew was estimated at the same time as the stability and control derivatives (described next) and did not exhibit any correlation issues. Overall, this process was a simple and effective way for synchronizing the data and enforcing consistency of the response measurements which improved the data quality. Although time skew and scale factor errors were small, they affected the parameter estimation results. In particular, the stability and control derivatives pertaining to the structural modes were sensitive to the time delay values.

Once the data were corrected for time skews and scale factor errors, output error parameter estimation was applied to determine estimates of the non-dimensional stability and control derivatives and the input time skew. Starting values for the model parameters in the nonlinear optimization were obtained from an aeroelastic panel code model of the X-56A using finite differences. The model structure (the subset of possible stability and control derivatives that can be accurately estimated using the data) was adjusted using trial and error until the parameter estimates seemed adequate, standard errors were reduced, fits to data were close, and the estimator converged quickly. This process was tedious and time consuming, but previous experience with this aircraft, insight using other 
analyses $[17,18]$, modifications to the output-error code to increase flexibility in choosing the model structure, and reduction of the output-error optimization problem helped.

For this analysis, structural parameters such as the mode shapes and in-vacuo frequencies were substituted from the FEM and only non-dimensional stability and control derivatives and the input time skew were estimated. If structural parameters were not available, as in many flight tests, they could also be estimated from the flight data. However, only the equivalent structural damping and frequency (for example, the combined $Q_{\eta_{\eta_{7}}}-\omega_{7}^{2}$ or $Q_{7_{\eta_{7}}}-2 \zeta_{7} \omega_{7}$ terms) could be identified in this case and maneuvers at different dynamic pressures would be required to separate the air-off and air-on contributions. Similarly, the mode shapes (for example, $\phi_{7_{z}}^{\text {soa }}$ ) could be identified, up to a scale factor, but these identified values would pertain to the air-on mode shapes and not correspond to the in-vacuo modes used as basis shapes to parameterize the structural deformations. Having accurate estimates of the structural parameters therefore allows more insight into physical parameters and creates a model with a larger range of applicability.

Fits of the model outputs to measured output data are shown in Figure 5. The x-axes span the bandwidth of the excitation, whereas all the y-axes have different ranges. The short period, SW1B, and SW1T modes are clearly present in the data. There was some excitation of the SWL mode in the higher frequencies. The SFA mode did not have much participation in these output measurements. Overall, the model outputs fit the data well over the entire excitation bandwidth. There was some mismatch at low frequencies near the short period mode in $\alpha^{v n}, a_{z}^{\text {egi }}$, and $a_{z}^{c f}$ and at higher frequencies in $a_{z}^{\text {sma }}$ and $a_{z}^{\text {sof }}$. These errors could be due to low-frequency coupling with neglected structural dynamics, unsteady aerodynamics, or other factors. The low-frequency mismatches could also be due in part to errors in the assumed displacement mode shapes of the structure at these sensors obtained from the FEM. However, in general these were good fits to the data and the estimator converged quickly from the starting values.

Estimates of non-dimensional stability and control derivatives are shown in Figure 6. Markers in blue squares were from a static wind tunnel test using a scaled half-span model of the X-56A. Prior estimates were not available for the angular rate derivatives or aeroelastic derivatives from these sources. Estimates shown as green diamond markers were obtained from the aeroelastic panel code model of the X-56A in the 50\% fuel configuration using finite differences. Estimates identified using output error in the frequency domain are shown as red circles, where the error bounds indicate the estimated $\pm 2 \sigma$ standard errors. The parameters $C_{Z_{q}}, C_{Q_{7_{\alpha}}}, C_{Q_{9_{\alpha}}}$, and $C_{Q_{9_{\eta}}}$ were not statistically significant, as indicated by the size of their standard errors, and were removed from the model structure. The other parameters, in general, had moderate agreement with the other predictions and had adequate standard error levels. The control derivatives were very close to the predicted values. The angle of attack derivatives had good agreement. The pitch rate derivatives exhibited larger amounts of variation between the estimates. These derivatives are weaker and are typically more difficult to estimate. Improvements could be made with a more refined data compatibility analysis and by installing additional high-rate gyroscopes in the aircraft. 

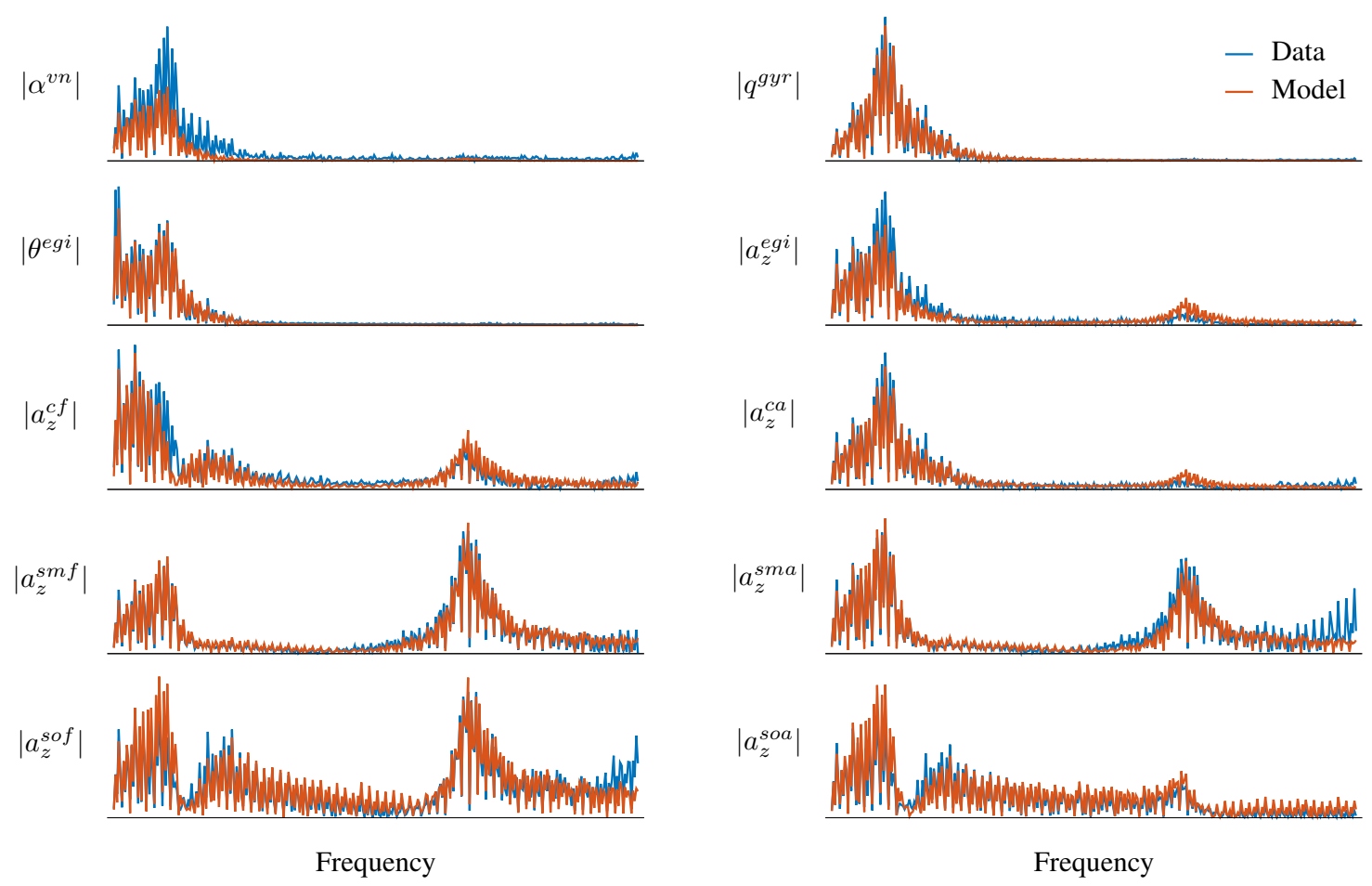

Figure 5. Model fits to Fourier transforms of measured outputs using output error.

A second maneuver was used to test the predictive capability of the model. The validation maneuver, data for which are shown in Figure 7, involved a different type of excitation input and was conducted during a subsequent flight at a different altitude and weight. The average fuel weight for the validation maneuver was $64 \%$, which was heavier than $32 \%$ used in the identification case and changed the aeroelastic properties of the airplane. The first column of plots in Figure 7 shows the control surface deflections. A doublet was performed on the $\delta_{w f 4 s}$ surface targeting the SW1B mode, although the short period and SW1T responses are also present in the response data. A control system was running and some feedback is present in the control surface deflections. The measured output responses are shown in blue in the second and third columns. The red time histories are the outputs of the identified linear model, driven using the measured control surface deflections. Although there are some differences between the model outputs and the measurements, the output of the model was in general close to the measured flight test data, thereby validating that the identified model captures the salient features of the response. The availability of FEM/GVT structural data and the identification of non-dimensional aeroelastic derivatives facilitated using a validation maneuver from a different flight condition than that used for identification. 


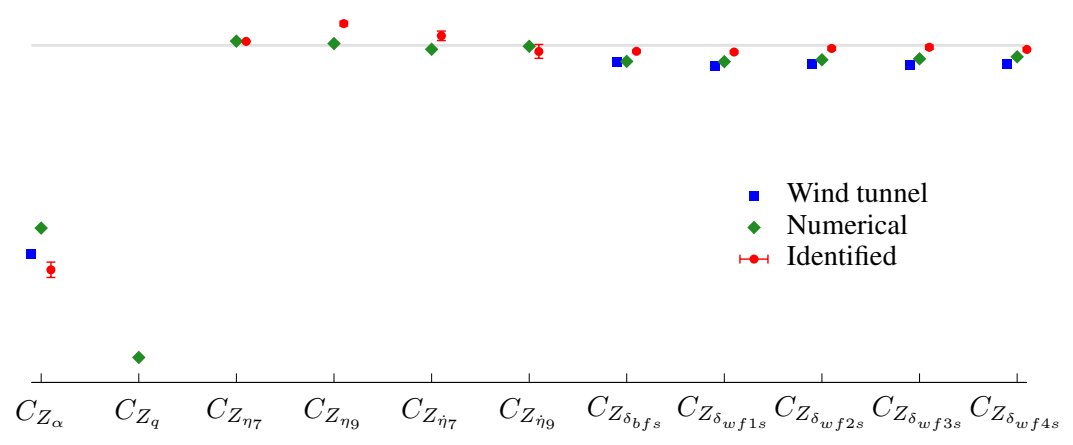

(a)

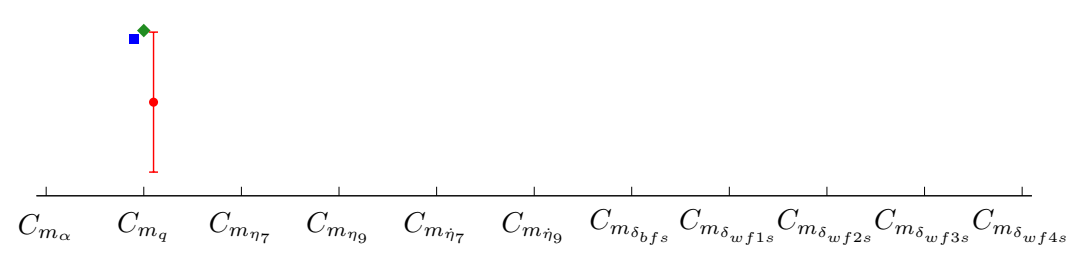

(b)

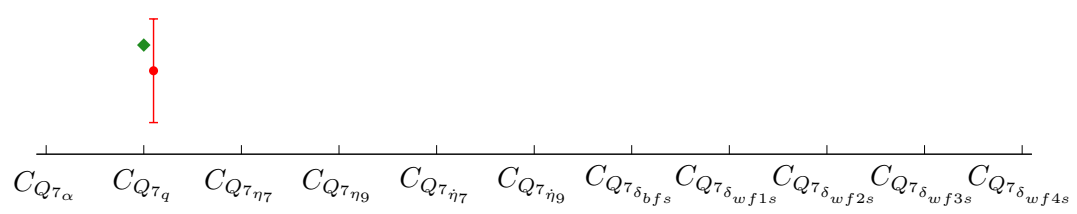

(c)

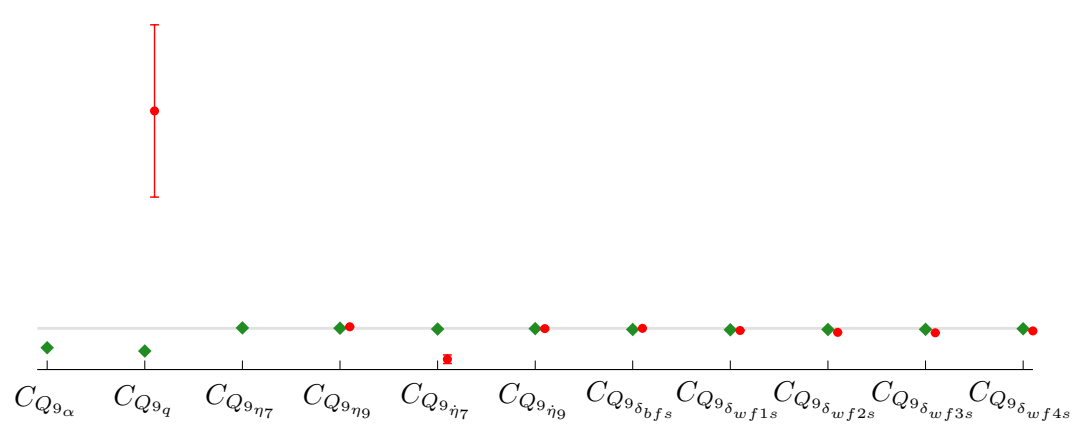

(d)

Figure 6. Estimated stability and control derivatives identified from wind tunnel tests with a scaled half-span model (blue), an aeroelastic panel code (green), and flight test data (red): (a) vertical force $C_{Z}$ derivatives; (b) pitching moment $C_{m}$ derivatives; (c) first symmetric wing bending $C_{Q_{7}}$ derivatives; and (d) first symmetric wing torsion $C_{Q_{9}}$ derivatives. 


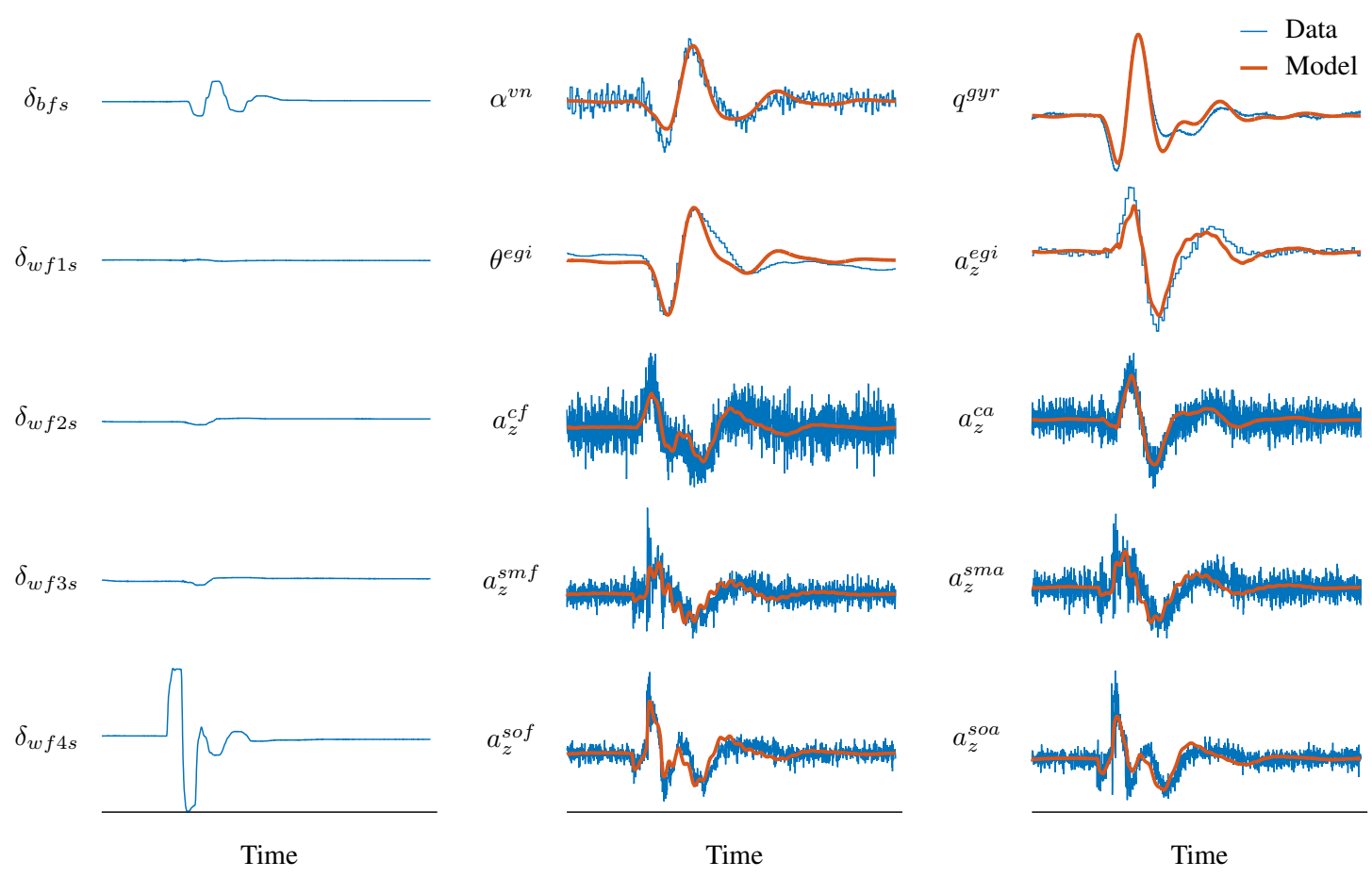

Figure 7. Model validation data (Flight 19, FTA 6232, 64\% fuel, 82\% flutter speed).

Figure 8 shows eigenvector phasor diagrams for the identified model. Drawn in polar coordinates, these diagrams illustrate how much and with what phasing each state participates in the modal response. Although each mode contains contributions from all states, only the most significant states are noted in these diagrams. Eigenvectors associated with the structural modes are still referred to as SW1B and SW1T here, although these are similar to but technically different from the in-vacuo vibration modes due to the aerodynamic interactions [5]. Figure 8a is the elastic short period mode. The normal phasing between $\alpha$ and $q$ is present, but the elastic states $\dot{\eta}_{7}, \eta_{7}$, and $\dot{\eta}_{9}$ play a large role in the modal response and indicate the large degree of flexibility in this aircraft. Note that the states $\alpha$ and $q$ in these phasor diagrams are different than the outputs $\alpha^{v n}$ and $q^{g y r}$ defined in Equations (17) and (18). For the SW1B mode shown in Figure 8b, the response is mainly due to $\dot{\eta}_{7}$ with smaller contributions due to $\eta_{7}$ and $\eta_{9}$. Similarly, for the SW1T mode shown in Figure 8c, the response is mainly due to $\dot{\eta}_{9}$ with smaller contributions due to $\eta_{7}$ and $\eta_{9}$.

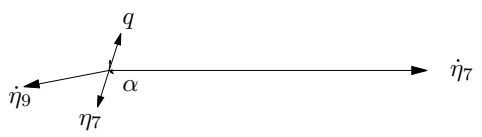

(a)

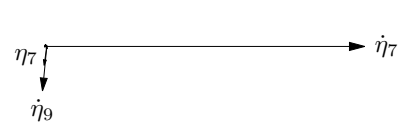

(b)

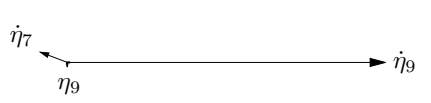

(c)

Figure 8. Eigenvector phasor diagrams for the identified model: (a) short period (SP) mode; (b) first symmetric wing bending (SW1B) mode; and (c) first symmetric wing torsion (SW1T) mode.

Bode plots showing the frequency responses from the $\delta_{w f 4 s}$ input to the measured pitch rate at the gyroscope $q^{g y r}$ are shown in Figure 9. The frequency response using the aeroelastic panel code, truncated to include only the short period, SW1B, and SW1T modes, is shown in green, whereas the identified model is shown in red. The frequency response for the identified model is similar to the aeroelastic panel code prediction, but has larger magnitudes near the short period and SW1B modes. The faster roll-off of the model after the SW1B mode exhibited in the identified response was corroborated by a separate analysis using frequency responses [18]. 


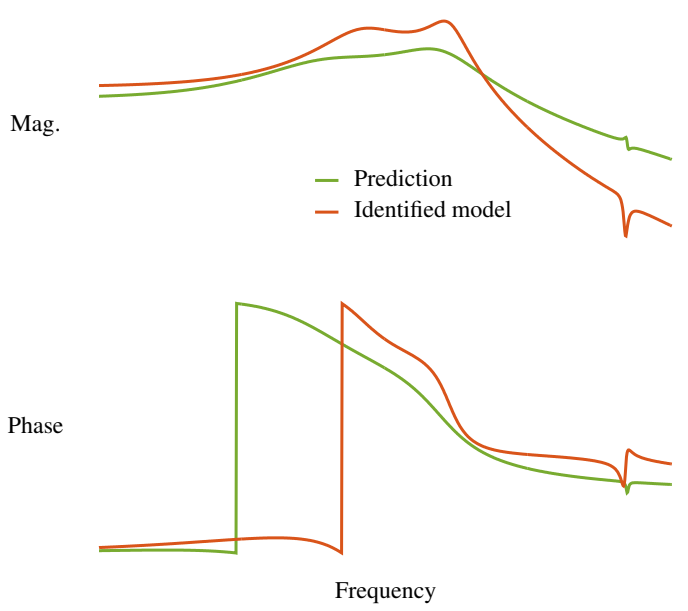

Figure 9. Bode plot of the $q^{g y r} / \delta_{w f 4 s}$ frequency response.

Figure 10 shows variation in the modal characteristics of the identified model as airspeed and dynamic pressure are increased. In Figure 10a, the dynamic-pressure root locus shows the migration of eigenvalues in the complex plane as dynamic pressure increases from about $15 \%$ of the flutter speed (green) to about $115 \%$ of the flutter speed, with all other model parameters (such as altitude, fuel weight, etc.) held constant. Again, SW1B and SW1T refer to the aeroelastic modes, which are technically different than the genesis vibration modes obtained when $\bar{q}=0$. In Figure 10b, the frequency and damping ratio of the eigenvalues are shown as airspeed increases over the same range. These plots indicate that as the X-56A flies faster, the aeroelastic SW1B and SW1T modes decrease slightly in frequency and increase in damping ratio, remaining stable, whereas the short period mode increases in frequency and decreases in damping ratio, becoming unstable. It was expected from other numerical modeling predictions that the short period and SW1B would coalesce at higher dynamic pressures; however, the SW1B mode was expected to become unstable, rather than the short period mode. Recent flight tests at airspeeds near and beyond the flutter speed have corroborated that the short period mode becomes unstable, as shown in Figure 10a.

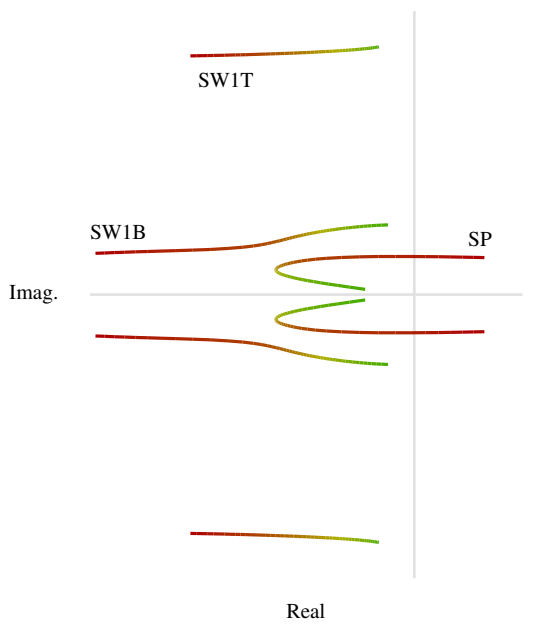

(a)

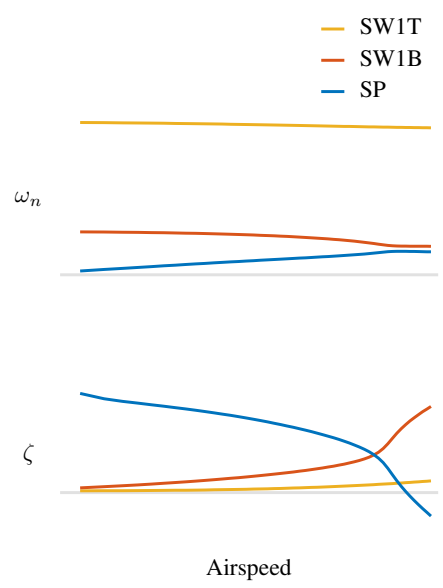

(b)

Figure 10. Variation in modal characteristics of the identified model with airspeed and dynamic pressure: (a) dynamic-pressure root locus of the identified model, with dynamic pressure increasing linearly from about $15 \%$ of the flutter speed (green) to about $115 \%$ of the flutter speed (red); and (b) frequency and damping ratio of eigenvalues for the identified model over the same airspeed range. 


\section{Conclusions}

In this paper, the identification of aeroelastic models for the X-56A airplane longitudinal dynamics from flight test data is discussed. The excitations for the maneuver were orthogonal phase-optimized multisine inputs, which perturbed control surface pairs at the same time but in different ways while a feedback control system was active. The Waszak-Schmidt aeroelastic flight dynamics model, an extension of traditional flight dynamics models using quasi-steady stability and control derivatives, was used for identification. Parameter estimation was performed using the output-error approach to match Fourier transforms of measured output response data. A validation maneuver was used to demonstrate the predictive capability of the identified model under different conditions, and modal characteristics of the identified model were explored. Practical aspects of the experiment design and system identification analysis for flexible aircraft are discussed throughout the paper.

The main findings of this paper can be summarized as follows:

1. Orthogonal phase-optimized multisines are effective inputs for efficiently exciting aeroelastic aircraft with multiple control effectors using a specified frequency bandwidth and power spectra for system identification.

2. Application of output error parameter estimation to determine non-dimensional stability and control derivatives that best match model outputs to Fourier transforms of measured output data was a successful approach for identifying an aeroelastic model of the X-56A from flight test data.

3. The Waszak-Schmidt aeroelastic flight dynamics model was useful for system identification and was a good representation of the observed flight dynamics at different airspeeds and fuel weights.

The orthogonal phase-optimized multisine inputs proved a good input for exciting the X-56A aircraft for aeroelastic identification. The inputs were run simultaneously on multiple control surfaces, which facilitated the identification of the entire model from a single maneuver with a relatively short time duration. Although a control system was running, control surface deflections exhibited low pair-wise correlations and were of good quality for modeling. Arbitrary design of the excitation bandwidth and power spectra facilitated targeting of specific modal responses in the output data for modeling.

The estimation of model parameters using output error to match Fourier transforms of measured response data had several advantages. Output error is based on maximum likelihood estimation, which gives a theoretical justification to the estimated parameters and uncertainties. Furthermore, it includes relevant simplifications and can incorporate a large amount of data measured from multiple sensors. Analysis using frequency-domain data reduces the number of data points to process and the number of unknown model parameters to estimate, and makes it computationally more efficient to solve the equations of motion for each iteration of the optimization. In general, the frequency-domain approach also illuminates modal responses, which are separated in frequency.

Drawbacks of the estimation approach are that the analysis is restricted to linear models, iterations are needed in the optimization from good starting values for the model parameters, and changes to the model structure can be cumbersome to implement. The results of this identification were intended for analysis and control design, and the maneuver involved small perturbations about a reference condition, so linear models were adequate for the identification. Starting values were obtained from a separate analysis using an aeroelastic panel code model. Convergence of the estimator was attained relatively quickly by simplifying the estimation problem and using frequency-domain data. Determining model structure, however, was tedious and time-consuming using this approach due to the large number of possible model parameters. Recent work with the X-56A using the equation-error approach, where statistical metrics can be applied to automatically select the aerodynamic model structure, and using frequency responses, where the model structure can be inferred from Bode plots, was leveraged in choosing the aeroelastic derivatives to estimate.

The Waszak-Schmidt aeroelastic flight dynamics model was an appropriate choice for system identification using the X-56A airplane. The model structure was relatively simple but able to capture 
the effects of aeroelasticity, including flutter, and was based on the familiar quasi-steady stability and control derivatives traditionally used for flight dynamics models. Because in-vacuo vibration modes parameterized the structural deformations and because data from a finite element model and ground vibration test were available, non-dimensional aeroelastic stability and control derivatives were identified. This allowed a single set of coefficients, obtained below flutter speeds, to predict aircraft dynamic behavior at higher speeds including the aeroelastic instability observed in flight.

Overall, the approach presented in this paper was an efficient, effective, and successful method for identifying aeroelastic flight dynamics models from measured flight test data. The results are representative of flight in several conditions, including those that result in aeroelastic instabilities such as flutter. The models identified can be used to refine simulation models and to design feedback control laws such as for active flutter suppression or gust load alleviation.

Author Contributions: Conceptualization, M.J.B. and J.A.G.; Methodology, M.J.B. and J.A.G.; Formal analysis, J.A.G.; Data curation, M.J.B.; Writing, M.J.B. and J.A.G.

Funding: This research was support by the NASA Advanced Air Transport Technology (AATT) Project.

Acknowledgments: The efforts of the X-56A team at NASA Armstrong Flight Research Center (AFRC) are gratefully acknowledged. Numerical predictions of stability and control derivatives were provided by Jeffrey Ouellette. Low-order models identified from flight data that contributed to understanding the identified model were provided by Christopher Miller and Jacob Schaefer. Discussions with Christopher Miller, Jeffrey Ouellette, Jacob Schaefer, and the other X-56A team members are appreciated.

Conflicts of Interest: The authors declare no conflict of interest.

\section{Abbreviations}

\begin{tabular}{|c|c|}
\hline \multicolumn{2}{|c|}{ Symbols } \\
\hline $\bar{c}$ & mean aerodynamic chord, $\mathrm{ft}$ \\
\hline $\operatorname{cov}[\cdot]$ & covariance \\
\hline$\partial$ & partial derivative \\
\hline$E[]$. & expectation operator \\
\hline$g$ & gravitational acceleration, $\mathrm{ft} / \mathrm{s}^{2}$ \\
\hline II & identity matrix \\
\hline$I_{y y}$ & pitch moment of inertia, slug- $\mathrm{ft}^{2}$ \\
\hline j & imaginary number, $=\sqrt{-1}$ \\
\hline$m$ & aircraft mass, slug \\
\hline$q$ & pitch rate, $\mathrm{rad} / \mathrm{s}$ \\
\hline $\bar{q}$ & dynamic pressure, $\mathrm{lbf} / \mathrm{ft}^{2}$ \\
\hline$\Re$ & real part \\
\hline$S$ & wing reference area, $\mathrm{ft}^{2}$ \\
\hline$t$ & time, s \\
\hline$V$ & true airspeed, $\mathrm{ft} / \mathrm{s}$ \\
\hline$X_{T}$ & longitudinal thrust component, $\mathrm{lbf}$ \\
\hline \multicolumn{2}{|c|}{ Greek } \\
\hline$\alpha$ & angle of attack, rad \\
\hline$\Delta$ & perturbation \\
\hline$\delta$ & control surface deflection, rad \\
\hline$\eta$ & modal displacement \\
\hline$\theta$ & pitch angle, rad \\
\hline$\sigma$ & standard deviation \\
\hline$\omega$ & frequency, rad/s \\
\hline
\end{tabular}




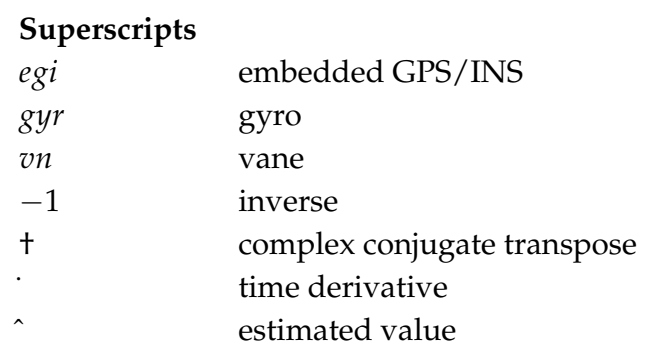

$\begin{array}{ll}\text { Acronyms } & \\ \text { SFA } & \text { symmetric fore-aft } \\ \text { SP } & \text { short period } \\ \text { SW1B } & \text { first symmetric wing bending } \\ \text { SW1T } & \text { first symmetric wing torsion } \\ \text { SWL } & \text { symmetric winglet lateral }\end{array}$

\section{References}

1. Ryan, J.; Bosworth, J.; Burken, J.; Suh, P. Current and Future Research in Active Control of Lightweight, Flexible Structures Using the X-56A Aircraft. In Proceedings of the 52nd Aerospace Sciences Meeting, National Harbor, MD, USA, 13-17 January 2014.

2. Beranek, J.; Nicolai, L.; Buonanno, M.; Atkinson, C.; Holm-Hansen, B.; Flick, P. Conceptual Design of a Multi-utility Aeroelastic Demonstrator. In Proceedings of the 13th AIAA/ISSMO Multidisciplinary Analysis Optimization Conference, Fort Worth, TX, USA, 13-15 September 2010.

3. Waszak, M.; Schmidt, D. Flight Dynamics of Aeroelastic Vehicles. J. Aircr. 1988, 25, 563-571. [CrossRef]

4. Waszak, M.; Buttrill, C.; Schmidt, D. Modeling and Model Simplification of Aeroelastic Vehicles: An Overview; Technical Report TM-107691; NASA: Hampton, VA, USA, 1992.

5. Schmidt, D. Modern Flight Dynamics; McGraw-Hill: New York, NY, USA, 2012.

6. Schmidt, D. MATLAB-Based Flight Dynamics and Flutter Modeling of a Flexible Flying-Wing Research Drone. J. Aircr. 2016, 53, 1045-1055. [CrossRef]

7. Schmidt, D. Stability Augmentation and Active Flutter Suppression of a Flexible Flying-Wing Drone. J. Guid. Control Dyn. 2016, 39, 409-422. [CrossRef]

8. McRuer, D.; Ashkenas, I.; Graham, D. Aircraft Dynamics and Automatic Control; Princeton University Press: Princeton, NJ, USA, 1973.

9. Acree, C.; Tischler, M. Determining XV-15 Aeroelastic Models from Flight Data with Frequency-Domain Methods; Technical Report TP-3330; NASA: Moffett Field, CA, USA, 1993.

10. Theodore, C.; Ivler, C.; Tischler, M.; Field, E.; Neville, R.; Ross, H. System Identification of Large Flexible Transport Aircraft. In Proceedings of the AIAA Atmospheric Flight Mechanics Conference and Exhibit, Honolulu, HI, USA, 18-21 August 2008.

11. Tischler, M.; Remple, R. Aircraft and Rotorcraft System Identification: Engineering Methods with Flight Test Examples, 2nd ed.; AIAA: Reston, VA, USA, 2012.

12. Juhasz, O.; Tischler, M.; Hagerott, S.; Staples, D.; Fuentealba, J. A Coupled Lateral/Directional Flight Dynamics and Structural Model for Flight Control Applications. In Proceedings of the Modeling and Simulation Technologies Conference, Kissimmee, FL, USA, 5-9 January 2015.

13. Powers, B. Structural Dynamic Model Obtained From Flight for Use With Piloted Simulation and Handling Qualities Analysis; Technical Report TM-4747; NASA: Edwards, CA, USA, 1996.

14. de Oliveira Silva, B.; Mönnich, W. System Identification of Flexible Aircraft in Time Domain. In Proceedings of the Atmospheric Flight Mechanics Conference, Baltimore, MD, USA, 13-16 August 2012.

15. Pfifer, H.; Danowsky, B. System Identification of a Small Flexible Aircraft. In Proceedings of the AIAA Atmospheric Flight Mechanics Conference, San Diego, CA, USA, 4-8 January 2016.

16. Danowsky, B.; Schmidt, D.; Pfifer, H. Control-Oriented System and Parameter Identification of a Small Flexible Flying-Wing Aircraft. In Proceedings of the Atmospheric Flight Mechanics Conference, Grapevine, TX, USA, 9-13 January 2017. 
17. Grauer, J.; Boucher, M. Real-Time Parameter Estimation for Flexible Aircraft. In Proceedings of the Atmospheric Flight Mechanics Conference, Atlanta, GA, USA, 25-29 June 2018.

18. Grauer, J.; Boucher, M. Identification of Bare-Airframe Dynamics from Closed-Loop Data Using Multisine Inputs and Frequency Responses. In Proceedings of the AIAA Scitech 2019 Forum, San Diego, CA, USA, 7-11 January 2019.

19. Livne, E. Aircraft Active Flutter Suppression: State of the Art and Technology Maturation Needs. J. Aircr. 2018, 55, 410-450. [CrossRef]

20. Morelli, E. System IDentification Programs for AirCraft (SIDPAC). Available online: http:/ / software.nasa. gov (accessed on 1 November 2018).

21. Pak, C.; Truong, S. Creating a Test-Validated Finite-Element Model of the X-56A Aircraft Structure. J. Aircr. 2015, 52, 1644-1667. [CrossRef]

22. Morelli, E.; Klein, V. Aircraft System Identification: Theory and Practice, 2nd ed.; Sunflyte: Williamsburg, VA, USA, 2016.

23. Steers, S.; Iliff, K. Effects of Time-Shifted Data on Flight-Determined Stability and Control Derivatives; Technical Report TN D-7830; NASA: Edwards, CA, USA, 1975.

24. Morelli, E. Multiple Input Design for Real-Time Parameter Estimation in the Frequency Domain. In Proceedings of the 13th IFAC Conference on System Identification, Rotterdam, The Netherlands, 27-29 August 2003; Volume 36, pp. 639-644.

25. Morelli, E. Flight-Test Experiment Design for Characterizing Stability and Control of Hypersonic Vehicles. J. Guid. Control Dyn. 2009, 32, 949-959. [CrossRef]

26. Grauer, J. Output Measurement Equations for Flexible Aircraft Flight Dynamics; Technical Report TM-2018-220102; NASA: Hampton, VA, USA, 2018.

27. Klein, V. Aircraft Parameter Estimation in Frequency Domain. In Proceedings of the Atmospheric Flight Mechanics Conference, Palo Alto, CA, USA, 7-9 August 1978.

28. Morelli, E. High Accuracy Evaluation of the Finite Fourier Transform Using Sampled Data; Technical Report TM-110340; NASA: Hampton, VA, USA, 1997.

29. Morelli, E. Dynamic Modeling from Flight Data with Unknown Time Skews. J. Guid. Control Dyn. 2017, 40, 2083-2091. [CrossRef]

(C) 2019 United States Government as represented by the Administrator of the National Aeronautics and Space Administration. No copyright is claimed in the United States under Title 17, U.S. Code. All Other Rights Reserved; Licensee MDPI, Basel, Switzerland. This article is an open access article distributed under the terms and conditions of the Creative Commons Attribution (CC BY) license (http://creativecommons.org/licenses/by/4.0/). 